TAIWANESE JOURNAL OF MATHEMATICS

Vol. 16, No. 6, pp. 2203-2238, December 2012

This paper is available online at http://journal.taiwanmathsoc.org.tw

\title{
BOUNDEDNESS OF MULTILINEAR COMMUTATORS OF CALDERÓN-ZYGMUND OPERATORS ON ORLICZ SPACES OVER NON-HOMOGENEOUS SPACES
}

\author{
Xing $\mathrm{Fu}$, Dachun Yang* and Wen Yuan
}

\begin{abstract}
Let $(\mathcal{X}, d, \mu)$ be a metric measure space satisfying both the upper doubling and the geometrically doubling conditions. In this paper, the authors prove that multilinear commutators of Calderon-Zygmund operators with $\operatorname{RBMO}(\mu)$ functions are bounded on Orlicz spaces, especially, on $L^{p}(\mu)$ with $p \in(1, \infty)$. The weak type endpoint estimate of multilinear commutators of Calderón-Zygmund operators with Orlicz type functions in $\operatorname{Osc}_{\exp L^{r}}(\mu)$ for $r \in[1, \infty)$ is also presented.
\end{abstract}

\section{INTRODUCTION}

The classical theory of singular integrals has been well developed into a large branch of analysis on spaces of homogeneous type in the sense of Coifman and Weiss $[5,6]$. Recall that a metric space $(\mathcal{X}, d)$ equipped with a nonnegative measure $\mu$ is called a space of homogeneous type if $(\mathcal{X}, d, \mu)$ satisfies the measure doubling condition: there exists a positive constant $C_{\mu}$ such that, for any ball $B(x, r):=\{y \in \mathcal{X}: d(x, y)<r\}$ with $x \in \mathcal{X}$ and $r \in(0, \infty), \mu(B(x, 2 r)) \leq C_{\mu} \mu(B(x, r))$. This measure doubling condition plays an important role in the classical Calderón-Zygmund theory.

However, in recent years, many results in the classical theory of Calderon-Zygmund operators on $\mathbb{R}^{d}$ have been proved still valid with the Lebesgue measure $m$ replaced by a Radon measure $\mu$ satisfying the polynomial growth condition (see, for example, $[22,23,24,25,26,27,17,3,7,8])$. Recall that a Radon measure $\mu$ on $\mathbb{R}^{d}$ is said

Received March 11, 2012, accepted April 6, 2012.

Communicated by Der-Chen Chang.

2010 Mathematics Subject Classification: Primary 47B47; Secondary 42B20, 42B35, 43A99.

Key words and phrases: Multilinear commutator, Calderón-Zygmund operator, Non-homogeneous space, Orlicz space.

This work is supported by the 2010 Joint Research Project Between China Scholarship Council and German Academic Exchange Service (PPP) (Grant No. LiuJinOu [2010]6066).

Dachun Yang is supported by the National Natural Science Foundation (Grant No. 11171027) of China and Program for Changjiang Scholars and Innovative Research Team in University of China.

Wen Yuan is supported by the National Natural Science Foundation (Grant No. 11101038) of China.

*Corresponding author. 
to satisfy the polynomial growth condition, if there exist positive constants $C_{0}$ and $\kappa \in(0, d]$ such that, for all $x \in \mathbb{R}^{d}$ and $r \in(0, \infty)$,

$$
\mu(B(x, r)) \leq C_{0} r^{\kappa},
$$

where $B(x, r):=\left\{y \in \mathbb{R}^{d}:|y-x|<r\right\}$. Tolsa [22,24] introduced the Hardy space $H^{1}(\mu)$ and its dual, the space of functions with regularized bounded mean oscillation, $\operatorname{RBMO}(\mu)$, with respect to $\mu$ as in (1.1), and proved that Calderón-Zygmund operators are bounded from $H^{1}(\mu)$ to $L^{1}(\mu)$. Later, Chen, Meng and Yang [3] showed that Calderon-Zygmund operators are bounded on $H^{1}(\mu)$. Nazarov, Treil and Volberg [17] established the $T b$ theorem in this setting. The analysis on such non-doubling spaces has been proved by Tolsa in $[25,26,27]$ to play a striking role in solving the longstanding open Painlevé's problem and Vitushkin's conjecture.

However, as pointed out by Hytönen in [9], the measures satisfying (1.1) do not include the doubling measures as special cases. In [9], Hytönen introduced a new class of metric measure spaces satisfying the so-called geometrically doubling and the upper doubling conditions (see, respectively, Definitions 1.1 and 1.3 below). This new class of metric measure spaces, which are called non-homogeneous spaces, includes both the spaces of homogeneous type and metric spaces with polynomial growth measures as special cases. In this new setting, Hytönen [9] introduced the $\operatorname{RBMO}(\mu)$ space and established the corresponding John-Nirenberg inequality. Later, Bui and Duong [2], and Hytönen, Da. Yang and Do. Yang [12] independently introduced the atomic Hardy space $H^{1}(\mu)$ and proved that the dual space of $H^{1}(\mu)$ is $\operatorname{RBMO}(\mu)$. Lin and Yang [14] introduced and investigated the space $\operatorname{RBLO}(\mu)$, a subset of $\operatorname{RBMO}(\mu)$, and obtained the boundedness of the maximal Calderón-Zygmund operators from $L^{\infty}(\mu)$ into $\operatorname{RBLO}(\mu)$. Hytönen, Liu, Da. Yang and Do. Yang [10] and Liu, Da. Yang and Do. Yang [15] established some equivalent characterizations for the boundedness of Carderon-Zygmund operators on $L^{p}(\mu)$ with $p \in(1, \infty)$ and its endpoint boundedness.

In 1976, Coifman, Rochberg and Weiss [4] proved that the commutator $[b, T]$ of a Calderón-Zygmund operator $T$ with a function $b \in \mathrm{BMO}\left(\mathbb{R}^{d}\right)$ defined by

$$
[b, T](f)(x):=b(x) T(f)(x)-T(b f)(x), \quad x \in \mathbb{R}^{d},
$$

is bounded on $L^{p}\left(\mathbb{R}^{d}\right)$ for all $p \in(1, \infty)$. From then on, there appeared a lot of literatures on the boundedness of commutators on various function spaces over different underlying spaces, and their applications in the theory of partial differential equations and harmonic analysis. In particular, for a Radon measure $\mu$ on $\mathbb{R}^{d}$ satisfying the growth condition (1.1), Tolsa [22] established the boundedness on $L^{p}(\mu)$ with $p \in$ $(1, \infty)$ of commutators of Carderon-Zygmund operators with $\operatorname{RBMO}(\mu)$ functions. The $L^{p}(\mu)$-boundedness of multilinear commutators of Carderón-Zygmund operators with $\operatorname{RBMO}(\mu)$ functions or some Orlicz type functions were further obtained in [7]. In [2], on non-homogeneous spaces $(\mathcal{X}, d, \mu)$, the $L^{p}(\mu)$-boundedness of commutators 
of Carderón-Zygmund operators with $\operatorname{RBMO}(\mu)$ functions was also obtained under the additional assumption: there exists $m \in(0, \infty)$ such that

$$
\lambda(x, a r)=a^{m} \lambda(x, r) \text { for all } x \in \mathcal{X} \text { and } a, r \in(0, \infty),
$$

where $\lambda$ is the dominating function of the measure $\mu$ (see, for example, Definition 1.3 below).

As a generalization of $L^{p}\left(\mathbb{R}^{d}\right)$, the Orlicz space was originally introduced by Birnbaum-Orlicz in [1] and Orlicz in [18]. Since then, the theory of Orlicz spaces has been well developed and widely used in various branches of mathematics, for example, probability, statistics, potential theory, partial differential equations, and harmonic analysis (see, for example, [16, 20, 21]).

Let $(\mathcal{X}, d, \mu)$ be a non-homogeneous space in the sense of Hytönen [9]. In this paper, we prove that, without the additional assumption (1.2), the multilinear commutators of Calderon-Zygmund operators with $\operatorname{RBMO}(\mu)$ functions are bounded on some Orlicz spaces $L^{\Phi}(\mu)$, especially, on $L^{p}(\mu)$ with $p \in(1, \infty)$. This, even for the commutator, also essentially improves the result about the $L^{p}(\mu)$-boundedness of commutators obtained in [2]. We also establish a weak type estimate for multilinear commutators of Calderon-Zygmund operators with some Orlicz type functions in $\mathrm{Osc}_{\exp L^{r}}(\mu)$.

To state the main results of this paper, we first recall some necessary notions and notation. The following notions of geometrically doubling and upper doubling metric measure spaces were originally introduced by Hytönen [9] (see also [10, 15]).

Definition 1.1. A metric space $(\mathcal{X}, d)$ is called geometrically doubling if there exists some $N_{0} \in \mathbb{N}$ such that, for any ball $B(x, r) \subset \mathcal{X}$, there exists a finite ball covering $\left\{B\left(x_{i}, r / 2\right)\right\}_{i}$ of $B(x, r)$ such that the cardinality of this covering is at most $N_{0}$.

Remark 1.2. Let $(\mathcal{X}, d)$ be a metric space. In [9], Hytönen showed that the following statements are mutually equivalent:

(i) $(\mathcal{X}, d)$ is geometrically doubling.

(ii) For any $\epsilon \in(0,1)$ and any ball $B(x, r) \subset \mathcal{X}$, there exists a finite ball covering $\left\{B\left(x_{i}, \epsilon r\right)\right\}_{i}$ of $B(x, r)$ such that the cardinality of this covering is at most $N_{0} \epsilon^{-n}$, here and in what follows, $N_{0}$ is as in Definition 1.1 and $n:=\log _{2} N_{0}$.

(iii) For every $\epsilon \in(0,1)$, any ball $B(x, r) \subset \mathcal{X}$ contains at most $N_{0} \epsilon^{-n}$ centers of disjoint balls $\left\{B\left(x_{i}, \epsilon r\right)\right\}_{i}$.

(iv) There exists $M \in \mathbb{N}$ such that any ball $B(x, r) \subset \mathcal{X}$ contains at most $M$ centers $\left\{x_{i}\right\}_{i}$ of disjoint balls $\left\{B\left(x_{i}, r / 4\right)\right\}_{i=1}^{M}$.

Definition 1.3. A metric measure space $(\mathcal{X}, d, \mu)$ is called upper doubling if $\mu$ is a Borel measure on $\mathcal{X}$ and there exist a dominating function $\lambda: \mathcal{X} \times(0, \infty) \rightarrow(0, \infty)$ 
and a positive constant $C_{\lambda}$, depending on $\lambda$, such that for each $x \in \mathcal{X}, r \rightarrow \lambda(x, r)$ is non-decreasing and, for all $x \in \mathcal{X}$ and $r \in(0, \infty)$,

$$
\mu(B(x, r)) \leq \lambda(x, r) \leq C_{\lambda} \lambda(x, r / 2) .
$$

Remark 1.4. (i) Obviously, a space of homogeneous type is a special case of upper doubling spaces, with the dominating function $\lambda(x, r):=\mu(B(x, r))$. On the other hand, the Euclidean space $\mathbb{R}^{d}$ with any Radon measure $\mu$ as in (1.1) is also an upper doubling measure space by taking the dominating function $\lambda(x, r):=C_{0} r^{\kappa}$.

(ii) Let $(\mathcal{X}, d, \mu)$ be an upper doubling space and $\lambda$ a dominating function on $\mathcal{X} \times(0, \infty)$ as in Definition 1.3. It was proved in [12] that there exists another dominating function $\widetilde{\lambda}$ such that $\widetilde{\lambda} \leq \lambda, C_{\tilde{\lambda}} \leq C_{\lambda}$ and, for all $x, y \in \mathcal{X}$ with $d(x, y) \leq$ $r$

$$
\widetilde{\lambda}(x, r) \leq C_{\widetilde{\lambda}} \widetilde{\lambda}(y, r) .
$$

Thus, in what follows, we always assume that $\lambda$ satisfies (1.4).

We now recall the coefficient $\delta(B, S)$ introduced in [9], which is analogous to the quantity $K_{Q, R}$ introduced by Tolsa [22] (see also [23, 24]).

Definition 1.5. For any two balls $B \subset S$, define

$$
\delta(B, S):=1+\int_{2 S \backslash B} \frac{1}{\lambda\left(c_{B}, d\left(x, c_{B}\right)\right)} d \mu(x),
$$

where $c_{B}$ is the center of the ball $B$.

Though the measure doubling condition is not assumed uniformly for all balls in the space $(\mathcal{X}, d, \mu)$ satisfying the geometrically and upper doubling conditions, it was shown in [9] that there are still many balls which have the following $(\alpha, \beta)$-doubling property.

Definition 1.6. Let $\alpha, \beta \in(1, \infty)$. A ball $B \subset \mathcal{X}$ is called $(\alpha, \beta)$-doubling if $\mu(\alpha B) \leq \beta \mu(B)$.

To be precise, it was proved in [9, Lemma 3.2] that if a metric measure space $(\mathcal{X}, d, \mu)$ is upper doubling and $\alpha, \beta \in(1, \infty)$ satisfying $\beta>C_{\lambda}^{\log _{2} \alpha}=: \alpha^{\nu}$, then for any ball $B \subset \mathcal{X}$, there exists some $j \in \mathbb{Z}_{+}:=\mathbb{N} \cup\{0\}$ such that $\alpha^{j} B$ is $(\alpha, \beta)$ doubling. Moreover, let $(\mathcal{X}, d)$ be geometrically doubling, $\beta>\alpha^{n}$ with $n:=\log _{2} N_{0}$ and $\mu$ a Borel measure on $\mathcal{X}$ which is finite on bounded sets. Hytönen [9, Lemma 3.3] showed that for $\mu$-almost every $x \in \mathcal{X}$, there exist arbitrary small $(\alpha, \beta)$-doubling balls centered at $x$. Furthermore, the radius of these balls may be chosen to be of the form $\alpha^{-j} r$ for $j \in \mathbb{N}$ and any preassigned number $r \in(0, \infty)$. Throughout this paper, 
for any $\alpha \in(1, \infty)$ and ball $B$, the smallest $\left(\alpha, \beta_{\alpha}\right)$-doubling ball of the form $\alpha^{j} B$ with $j \in \mathbb{Z}_{+}$is denoted by $\widetilde{B}^{\alpha}$, where

$$
\beta_{\alpha}:=\max \left\{\alpha^{3 n}, \alpha^{3 \nu}\right\}+30^{n}+30^{\nu}=\alpha^{3 \max \{n, \nu\}}+30^{n}+30^{\nu} .
$$

In what follows, by a doubling ball we mean a $\left(6, \beta_{6}\right)$-doubling ball and $\widetilde{B}^{6}$ is simply denoted by $\widetilde{B}$.

Now we recall the following notion of $\operatorname{RBMO}(\mu)$ from [9].

Definition 1.7. Let $\rho \in(1, \infty)$. A function $f \in L_{\text {loc }}^{1}(\mu)$ is said to be in the space $\operatorname{RBMO}(\mu)$ if there exist a positive constant $C$ and, for any ball $B \subset \mathcal{X}$, a number $f_{B}$ such that

$$
\frac{1}{\mu(\rho B)} \int_{B}\left|f(x)-f_{B}\right| d \mu(x) \leq C
$$

and, for any two balls $B$ and $B_{1}$ such that $B \subset B_{1}$,

$$
\left|f_{B}-f_{B_{1}}\right| \leq C \delta\left(B, B_{1}\right) .
$$

The infimum of the positive constants $C$ satisfying both (1.6) and (1.7) is defined to be the $\operatorname{RBMO}(\mu)$ norm of $f$ and denoted by $\|f\|_{\operatorname{RBMO}(\mu)}$.

From [9, Lemma 4.6], it follows that the space $\operatorname{RBMO}(\mu)$ is independent of $\rho \in(1, \infty)$.

The following notion of Carlderón-Zygmund operators comes from [11].

Definition 1.8. A function $K \in L_{\text {loc }}^{1}((\mathcal{X} \times \mathcal{X}) \backslash\{(x, y): x=y\})$ is called a Calderon-Zygmund kernel if there exists a positive constant $C_{K}$, depending on $K$, such that

(i) for all $x, y \in \mathcal{X}$ with $x \neq y$,

$$
|K(x, y)| \leq C_{K} \frac{1}{\lambda(x, d(x, y))}
$$

(ii) there exist positive constants $\delta \in(0,1]$ and $c_{K}$, depending on $K$, such that, for all $x, \widetilde{x}, y \in \mathcal{X}$ with $d(x, y) \geq c_{K} d(x, \widetilde{x})$,

$$
|K(x, y)-K(\widetilde{x}, y)|+|K(y, x)-K(y, \widetilde{x})| \leq C_{K} \frac{[d(x, \widetilde{x})]^{\delta}}{[d(x, y)]^{\delta} \lambda(x, d(x, y))}
$$

A linear operator $T$ is called a Calderón-Zygmund operator with kernel $K$ satisfying (1.8) and (1.9) if, for all $f \in L^{\infty}(\mu)$ with bounded support and $x \notin \operatorname{supp} f$,

$$
T f(x):=\int_{\mathcal{X}} K(x, y) f(y) d \mu(y) .
$$


A new example of operators with kernel satisfying (1.8) and (1.9) is the so-called Bergman-type operator appearing in [28]; see also [11] for an explanation. Notice that (1.8) and (1.9) are more general than the conditions satisfied by the classical CalderónZygmund operators.

Let $\Phi$ be a convex Orlicz function on $[0, \infty)$, namely, a convex increasing function satisfying $\Phi(0)=0, \Phi(t)>0$ for all $t \in(0, \infty)$ and $\Phi(t) \rightarrow \infty$ as $t \rightarrow \infty$. Let

$$
a_{\Phi}:=\inf _{t \in(0, \infty)} \frac{t \Phi^{\prime}(t)}{\Phi(t)} \quad \text { and } \quad b_{\Phi}:=\sup _{t \in(0, \infty)} \frac{t \Phi^{\prime}(t)}{\Phi(t)} .
$$

We refer to [16] for more properties of $a_{\Phi}$ and $b_{\Phi}$.

The Orlicz space $L^{\Phi}(\mu)$ is defined to be the space of all measurable functions $f$ on $(\mathcal{X}, d, \mu)$ such that $\int_{\mathcal{X}} \Phi(|f(x)|) d \mu(x)<\infty$; moreover, for any $f \in L^{\Phi}(\mu)$, its Luxemburg norm in $L^{\Phi}(\mu)$ is defined by

$$
\|f\|_{L^{\Phi}(\mu)}:=\inf \left\{t \in(0, \infty): \int_{\mathcal{X}} \Phi(|f(x)| / t) d \mu(x) \leq 1\right\} .
$$

For any sequence $\vec{b}:=\left(b_{1}, \cdots, b_{k}\right)$ of functions, the multilinear commutator $T_{\vec{b}}$ of the Calderon-Zygmund operator $T$ and $\vec{b}$ is defined by setting, for all suitable functions $f$ and $x \in \mathcal{X}$,

$$
T_{\vec{b}} f(x):=\left[b_{k},\left[b_{k-1}, \cdots,\left[b_{1}, T\right] \cdots\right]\right] f(x),
$$

where

$$
\left[b_{1}, T\right] f(x):=b_{1}(x) T f(x)-T\left(b_{1} f\right)(x) .
$$

The first main result of this paper is the following boundedness of multilinear commutators on Orlicz spaces.

Theorem 1.9. Let $k \in \mathbb{N}, b_{i} \in \operatorname{RBMO}(\mu)$ for all $i \in\{1, \cdots, k\}$, $\Phi$ a convex Orlicz function satisfying that $1<a_{\Phi} \leq b_{\Phi}<\infty$. Assume that $T$ is a CalderonZygmund operator which is bounded on $L^{2}(\mu)$. Then the multilinear commutator $T_{\vec{b}}$ in (1.12) is bounded on Orlicz spaces $L^{\Phi}(\mu)$, namely, there exists a positive constant $C$ such that for all $f \in L^{\Phi}(\mu)$,

$$
\left\|T_{\vec{b}} f\right\|_{L^{\Phi}(\mu)} \leq C\left\|b_{1}\right\|_{\operatorname{RBMO}(\mu)} \cdots\left\|b_{k}\right\|_{\operatorname{RBMO}(\mu)}\|f\|_{L^{\Phi}(\mu)} .
$$

Remark 1.10. (i) Let $\Phi_{1}(t):=t^{p}$ for all $t \in(0, \infty)$ with $p \in(1, \infty)$. Then $\Phi_{1}$ is a convex Orlicz function with $a_{\Phi_{1}}=b_{\Phi_{1}}=p \in(1, \infty)$, and $L^{\Phi_{1}}(\mu)=L^{p}(\mu)$. In this case, Theorem 1.9 when $k=1$ also essentially improves [2, Theorem 7.6] by 
removing the additional assumption (1.2); moreover, if $\mathcal{X}=\mathbb{R}^{d}$ and $\mu$ is as in (1.1), then Theorem 1.9 is just [7, Theorem 2].

(ii) We remark that there exist non-trivial convex Orlicz functions satisfying the assumption of Theorem 1.9. For example, if $\Phi_{2}(t):=t^{p} \ln (e+t)$ for all $t \in[0, \infty)$ with $p \in(1, \infty)$, then $1<p=a_{\Phi_{2}} \leq b_{\Phi_{2}}<\infty$; if $\Phi_{3}(t):=t^{p} / \ln (e+t)$ for all $t \in[0, \infty)$ with $p \in(2, \infty)$, then $1<a_{\Phi_{3}} \leq b_{\Phi_{3}}=p<\infty$.

The endpoint counterpart of Theorem 1.9 is also considered in this paper. To this end, we first recall the following Orlicz type function space $\operatorname{Osc}_{\exp L^{r}}(\mu)$ (see, for example, Perez and Trujillo-González [19] for Euclidean spaces, and [7] for nondoubling measures).

In what follows, let $L_{\text {loc }}^{1}(\mu)$ be the space of all locally $\mu$-integrable functions on $\mathcal{X}$. For all balls $B$ and $f \in L_{\text {loc }}^{1}(\mu), m_{B}(f)$ denotes the mean value of $f$ on ball $B$, namely,

$$
m_{B}(f):=\frac{1}{\mu(B)} \int_{B} f d \mu .
$$

Definition 1.11. For $r \in[1, \infty)$, a function $f \in L_{\text {loc }}^{1}(\mu)$ is said to belong to the space $\operatorname{Osc}_{\exp L^{r}}(\mu)$ if there exists a positive constant $C_{1}$ such that

(i) for all balls $B$,

$$
\begin{aligned}
& \left\|f-m_{\widetilde{B}}(f)\right\|_{\exp L^{r}, B, \mu / \mu(2 B)} \\
:= & \inf \left\{\lambda \in(0, \infty): \frac{1}{\mu(2 B)} \int_{B} \exp \left(\frac{\left|f-m_{\widetilde{B}}(f)\right|}{\lambda}\right)^{r} d \mu \leq 2\right\} \leq C_{1},
\end{aligned}
$$

(ii) for all doubling balls $Q \subset R$,

$$
\left|m_{Q}(f)-m_{R}(f)\right| \leq C_{1} \delta(Q, R) .
$$

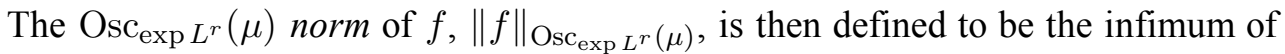
all positive constants $C_{1}$ satisfying (i) and (ii).

Remark 1.12. Obviously, for any $r \in[1, \infty), \operatorname{Osc}_{\exp L^{r}}(\mu) \subset \operatorname{RBMO}(\mu)$. Moreover, from [9, Corollary 6.3], it follows that $\operatorname{Osc}_{\exp L^{1}}(\mu)=\operatorname{RBMO}(\mu)$.

Now we state another main result of this paper.

Theorem 1.13. Let $k \in \mathbb{N}, r_{i} \in[1, \infty)$ and $b_{i} \in \mathrm{Osc}_{\exp } L^{r_{i}}(\mu)$ for $i \in\{1, \cdots, k\}$. Let $T$ and $T_{\vec{b}}$ be as in (1.10) and (1.12), respectively. If $T$ is bounded on $L^{2}(\mu)$, then there exists a positive constant $C$ such that, for all $\lambda \in(0, \infty)$ and all bounded functions $f$ with bounded support,

$$
\begin{aligned}
\mu\left(\left\{x \in \mathcal{X}:\left|T_{\vec{b}} f(x)\right|>\lambda\right\}\right) \leq & C \Phi_{1 / r}\left(\left\|b_{1}\right\|_{\mathrm{Osc}_{\exp L} r_{1}(\mu)} \cdots\left\|b_{k}\right\|_{\mathrm{Osc}_{\exp L_{k}} r_{k}(\mu)}\right) \\
& \times \int_{\mathcal{X}} \Phi_{1 / r}\left(\frac{|f(y)|}{\lambda}\right) d \mu(y),
\end{aligned}
$$

where $1 / r=1 / r_{1}+\cdots+1 / r_{k}$ and, for all $t \in(0, \infty)$ and $s \in(0, \infty), \Phi_{s}(t)=t \log ^{s}(2+t)$. 
Remark 1.14. Theorem 1.13 generalizes [7, Theorem 4] by taking $\mathcal{X}=\mathbb{R}^{d}$, $d(x, y)=|x-y|$ and $\mu$ being as in (1.1).

To prove Theorem 1.9, we first establish an interpolation theorem on Orlicz spaces in Theorem 2.2 below, which is obtained via a variant of the method for the classical Marcinkiewicz interpolation theorem and some properties of Orlicz functions. Let $\widetilde{\delta}(B, S)$ be the discrete version of $\delta(B, S)$, which was first introduced by Bui and Duong in [2] (see also (3.3) below). Via introducing a sharp maximal function $\widetilde{M}^{\#} f$, associated with $\widetilde{\delta}(B, S)$, in Definition 3.6 below and fully applying the properties of $\delta(B, S)$ and $\widetilde{\delta}(B, S)$ (see Lemmas 3.1 and 3.5 below), we prove in Theorem 3.10 below that, without the assumption (1.2), the commutators of Calderon-Zygmund operators and $\operatorname{RBMO}(\mu)$ functions are bounded on $L^{p}(\mu)$ for all $p \in(1, \infty)$. When (1.2) holds, this result was already obtained in [2, Theorem 7.6]. Comparing with the proof of $[2$, Theorem 7.6], the main new ingredients appearing in our approach used for the proof of Theorem 3.10 are that we replace the sharp maximal function $M^{\#} f$ in [2] by $\widetilde{M}^{\#} f$ here and that we observe that $\widetilde{\delta}(B, S)$ preserves most of the properties of $\delta(B, S)$ (see Lemma 3.5 below), which further induce a stronger version (see Theorem 3.8 below) of [2, Theorem 4.2] and hence lead us to avoid the assumption (1.2). Combining the aforementioned interpolation theorem on Orlicz spaces and the $L^{p}(\mu)$-boundedness of commutators in Theorem 3.10, we further show Theorem 1.9.

To establish the weak type endpoint estimate of multilinear commutators in Theorem 1.13, we need a generalized Hölder's inequality in the non-homogeneous setting (see Lemma 4.1 below), which is a generalization of [19, Lemma 2.3], and the CalderónZygmund decomposition in the non-homogeneous setting obtained by Bui and Duong [2].

The organization of this paper is as follows. In Section 2, we establish an interpolation theorem on Orlicz spaces. In Section 3, we show Theorem 1.9. Section 4 is devoted to the proof of Theorem 1.13.

Finally, we make some conventions on notation. Throughout the whole paper, $C$ stands for a positive constant which is independent of the main parameters, but it may vary from line to line. Constants with subscripts, such as $C_{0}$, do not change in different occurrences. Furthermore, we use $C(\rho, \alpha, \cdots)$ to denote a positive constant depending on the parameter $\rho, \alpha, \cdots$. For any ball $B$ and $f \in L_{\mathrm{loc}}^{1}(\mu), m_{B}(f)$ denotes the mean value of $f$ over $B$ as in (1.14); the center and the radius of $B$ are denoted, respectively, by $c_{B}$ and $r_{B}$. If $f \leq C g$, we then write $f \lesssim g$; if $f \lesssim g \lesssim f$, we then write $f \sim g$. For any subset $E$ of $\mathcal{X}$, we use $\chi_{E}$ to denote its characteristic function.

\section{AN InTERPolation ThEOREM}

In this section, we establish an interpolation theorem of Orlicz spaces, which plays a key role in the proof of Theorem 1.9.

We begin with some properties of the indices $a_{\Phi}$ and $b_{\Phi}$. 
Proposition 2.1. Let $\Phi$ be a convex Orlicz function on $[0, \infty), a_{\Phi}$ and $b_{\Phi}$ be as in (1.11).

(i) If $b_{\Phi}<\infty$, then $\Phi$ satisfies the $\nabla_{2}$ condition, namely, there exists a positive constant $C$ such that, for all $t \in(0, \infty), \Phi(2 t) \leq C \Phi(t)$.

(ii) If $b_{\Phi}<\infty$, then $\Phi(t) / t^{b_{\Phi}}$ is decreasing for $t \in(0, \infty)$. Moreover, for any given $\lambda \in[0,1]$ and $t \in(0, \infty), \Phi(\lambda t) \geq \lambda^{b_{\Phi}} \Phi(t)$.

(iii) $\Phi(t) / t^{a}$ is increasing for $t \in(0, \infty)$. Moreover, for any given $\lambda \in[1, \infty)$ and $t \in(0, \infty), \Phi(\lambda t) \geq \lambda^{a_{\Phi}} \Phi(t)$.

(iv) Let $1<p<a_{\Phi} \leq b_{\Phi}<q<\infty$. Then $\lim _{t \rightarrow 0} \frac{\Phi(t)}{t^{p}}=0$ and $\lim _{t \rightarrow \infty} \frac{\Phi(t)}{t^{q}}=0$.

\section{Proof.}

(i) By $b_{\Phi}<\infty$, we know that, for any $t \in(0, \infty), \frac{\Phi^{\prime}(t)}{\Phi(t)} \leq \frac{b_{\Phi}}{t}$; moreover, by the fact that any convex function on $[0, \infty)$ is absolutely continuous on every finite closed intervals of $[0, \infty)$, we see that

$$
\log \frac{\Phi(2 t)}{\Phi(t)}=\int_{t}^{2 t} \frac{\Phi^{\prime}(s)}{\Phi(s)} d s \leq \int_{t}^{2 t} \frac{b_{\Phi}}{s} d s=b_{\Phi} \log 2 .
$$

Thus, we have $\Phi(2 t) \leq 2^{b_{\Phi}} \Phi(t)$ for any $t \in(0, \infty)$. This shows (i).

(ii) For any given $t_{1}, t_{2} \in(0, \infty), t_{1} \leq t_{2}$, by the fundamental theorem of calculus, we see that

$$
\frac{\Phi\left(t_{2}\right)}{t_{2}^{b_{\Phi}}}-\frac{\Phi\left(t_{1}\right)}{t_{1}^{b_{\Phi}}}=\int_{t_{1}}^{t_{2}} \frac{s \Phi^{\prime}(s)-b_{\Phi} \Phi(s)}{s^{b_{\Phi}+1}} d s \leq 0 .
$$

Then $\Phi(t) / t^{b_{\Phi}}$ is decreasing for $t \in(0, \infty)$. Specially, for $\lambda \in(0,1]$ and $t \in(0, \infty), \frac{\Phi(t)}{t^{b} \Phi} \leq \frac{\Phi(\lambda t)}{(\lambda t)^{b_{\Phi}}}$, that is, $\Phi(\lambda t) \geq \lambda^{b_{\Phi}} \Phi(t)$, which completes the proof of (ii).

(iii) The proof of (iii) is similar to (ii). We omit the details.

(iv) For $t \in(0,1]$, since $\frac{\Phi(t)}{t^{a_{\Phi}}}$ is increasing on $t$, we then see that $\frac{\Phi(t)}{t^{a} \Phi} \leq \Phi(1)<\infty$. This, combined with $a_{\Phi}>p$, implies that

$$
\lim _{t \rightarrow 0} \frac{\Phi(t)}{t^{p}}=\lim _{t \rightarrow 0} t^{a_{\Phi}-p} \frac{\Phi(t)}{t^{a_{\Phi}}}=0
$$

For $t \in[1, \infty)$, since $\frac{\Phi(t)}{t^{a} \Phi}$ is decreasing on $t, \frac{\Phi(t)}{t^{b} \Phi} \leq \Phi(1)<\infty$. This, together with $b_{\Phi}<q$, further implies that

$$
\lim _{t \rightarrow \infty} \frac{\Phi(t)}{t^{q}}=\lim _{t \rightarrow \infty} t^{b_{\Phi}-q} \frac{\Phi(t)}{t^{b_{\Phi}}}=0,
$$

which completes the proof of (iv) and hence Proposition 2.1. 
In what follows, for a convex Orlicz function $\Phi:[0, \infty) \rightarrow[0, \infty)$, its inverse $\Phi^{-1}$ is defined by setting, for all $t \in[0, \infty)$,

$$
\Phi^{-1}(t):=\inf \{s \in(0, \infty): \Phi(s)>t\} .
$$

With these conclusions, we establish the following interpolation theorem.

Theorem 2.2. Let $\alpha \in[0,1), p_{i}, q_{i} \in(0, \infty)$ satisfy $1 / q_{i}=1 / p_{i}-\alpha$ for $i \in\{1,2\}$, $p_{1}<p_{2}$ and $T$ be a sublinear operator of weak type $\left(p_{i}, q_{i}\right)$ for $i \in\{1,2\}$. Then $T$ is bounded from $L^{\Phi}(\mu)$ to $L^{\Psi}(\mu)$, where $\Phi$ and $\Psi$ are convex Orlicz functions satisfying the following conditions: $1<p_{1}<a_{\Phi} \leq b_{\Phi}<p_{2}<\infty, 1<q_{1}<a_{\Psi} \leq b_{\Psi}<q_{2}<$ $\infty$ and, for all $t \in(0, \infty), \Psi^{-1}(t)=\Phi^{-1}(t) t^{-\alpha}$.

Proof. First, we show that $L^{\Phi}(\mu) \subset L^{p_{1}}(\mu)+L^{p_{2}}(\mu)$. To this end, for any given $\lambda \in(0, \infty)$, we decompose $f \in L^{\Phi}(\mu)$ as

$$
f(x)=f(x) \chi_{\{x \in \mathcal{X}:|f(x)|>\lambda\}}(x)+f(x) \chi_{\{x \in \mathcal{X}:|f(x)| \leq \lambda\}}(x)=: f^{\lambda}(x)+f_{\lambda}(x)
$$

for all $x \in \mathcal{X}$. For the sake of simplicity, we assume that $f \not \equiv 0$ on $\mathcal{X}$. Then we claim that $f^{\lambda} \in L^{p_{1}}(\mu)$ and $f_{\lambda} \in L^{p_{2}}(\mu)$. Indeed, by (i) and (iii) of Proposition 2.1, there exists a positive constant $C(\lambda)$, depending on $\lambda$, such that, for all $x \in \mathcal{X}$ satisfying $|f(x)|>\lambda$

$$
\left[\frac{|f(x)|}{\lambda}\right]^{a_{\Phi}} \leq \frac{\Phi(|f(x)| / \lambda)}{\Phi(1)} \leq C(\lambda) \frac{\Phi(|f(x)|)}{\Phi(1)}
$$

which, together with $p_{1}<a_{\Phi}$, implies that

$$
\begin{aligned}
& \int_{\mathcal{X}}\left|f^{\lambda}(x)\right|^{p_{1}} d \mu(x) \\
= & \int_{\{x \in \mathcal{X}:|f(x)|>\lambda\}}|f(x)|^{p_{1}} d \mu(x) \leq \int_{\{x \in \mathcal{X}:|f(x)|>\lambda\}} \frac{|f(x)|^{a_{\Phi}-p_{1}}}{\lambda^{a_{\Phi}}-p_{1}}|f(x)|^{p_{1}} d \mu(x) \\
\leq & C(\lambda) \frac{\lambda^{p_{1}}}{\Phi(1)} \int_{\mathcal{X}} \Phi(|f(x)|) d \mu(x)<\infty,
\end{aligned}
$$

namely, $f^{\lambda} \in L^{p_{1}}(\mu)$.

Now we show $f_{\lambda} \in L^{p_{2}}(\mu)$. By (i) and (ii) of Proposition 2.1, there exists a positive constant $C(\lambda)$, depending on $\lambda$, such that, for all $x \in \mathcal{X}$ satisfying $|f(x)| \leq \lambda$,

$$
\left[\frac{|f(x)|}{\lambda}\right]^{b_{\Phi}} \leq \frac{\Phi(|f(x)| / \lambda)}{\Phi(1)} \leq C(\lambda) \frac{\Phi(|f(x)|)}{\Phi(1)} .
$$

This, combined with $b_{\Phi}<p_{2}$, implies that 


$$
\begin{aligned}
\int_{\mathcal{X}}\left|f_{\lambda}(x)\right|^{p_{2}} d \mu(x) & =\int_{\{x \in \mathcal{X}:|f(x)| \leq \lambda\}}|f(x)|^{p_{2}} d \mu(x) \\
& \leq \lambda^{p_{2}-b_{\Phi}} \int_{\{x \in \mathcal{X}:|f(x)| \leq \lambda\}}|f(x)|^{b_{\Phi}} d \mu(x) \\
& \leq C(\lambda) \frac{\lambda^{p_{2}}}{\Phi(1)} \int_{\mathcal{X}} \Phi(|f(x)|) d \mu(x)<\infty,
\end{aligned}
$$

namely, $f_{\lambda} \in L^{p_{2}}(\mu)$, which proves the previous claim, and hence $L^{\Phi}(\mu) \subset L^{p_{1}}(\mu)+$ $L^{p_{2}}(\mu)$.

Next we show that $T$ is bounded from $L^{\Phi}(\mu)$ to $L^{\Psi}(\mu)$. To this end, let $u$ be a function on $[0, \infty)$ satisfying $u^{-1}(t)=\Psi^{-1}(\Phi(t))$ for all $t \in[0, \infty)$. Then $u^{-1}$ is nondecreasing function defined on $[0, \infty)$ such that $u^{-1}(t) \rightarrow 0$ as $t \rightarrow 0$ and $u^{-1}(t) \rightarrow \infty$ as $t \rightarrow \infty$. We also let $\sigma(f, \lambda):=\mu(\{x \in \mathcal{X}:|f(x)|>\lambda\})$. Then by the layer cake representation (see, for example, [13, Theorem 1.13]), we see that

$$
\begin{aligned}
\int_{\mathcal{X}} \Psi(|T f(x)|) d \mu(x)= & \int_{0}^{\infty} \sigma(T f, \lambda) d \Psi(\lambda) \\
\leq & \int_{0}^{\infty} \sigma\left(T f^{u(\lambda)}, \lambda / 2\right) d \Psi(\lambda) \\
& +\int_{0}^{\infty} \sigma\left(T f_{u(\lambda)}, \lambda / 2\right) d \Psi(\lambda)=: \mathrm{I}+\mathrm{II} .
\end{aligned}
$$

Since $T$ is of weak type $\left(p_{1}, q_{1}\right)$, we then see that

$$
\sigma\left(T f^{u(\lambda)}, \lambda / 2\right) \lesssim\left(\frac{2}{\lambda}\right)^{q_{1}}\left\|f^{u(\lambda)}\right\|_{L^{p_{1}(\mu)}}^{q_{1}},
$$

which, together with $p_{1}<q_{1}$ and Minkowski's inequality, implies that

$$
\begin{aligned}
& \mathrm{I}^{p_{1} / q_{1}} \\
& \lesssim\left.\int_{0}^{\infty}\left[\int_{\mathcal{X}} \lambda^{-p_{1}}|f(x)|^{p_{1}} \chi_{\{x \in \mathcal{X}:|f(x)|>u(\lambda)\}}(x) d \mu(x)\right]^{q_{1} / p_{1}} d \Psi(\lambda)\right\}^{p_{1} / q_{1}} \\
& \lesssim \int_{\mathcal{X}}\left[\int_{0}^{\infty} \lambda^{-q_{1}}|f(x)|^{q_{1}} \chi_{\{x \in \mathcal{X}:|f(x)|>u(\lambda)\}}(\lambda) d \Psi(\lambda)\right]^{p_{1} / q_{1}} d \mu(x) \\
& \lesssim \int_{\mathcal{X}}|f(x)|^{p_{1}}\left[\int_{0}^{u^{-1}(|f(x)|)} \lambda^{-q_{1}} d \Psi(\lambda)\right]^{p_{1} / q_{1}} d \mu(x) .
\end{aligned}
$$

By integration by parts, together with $u^{-1}(t) \rightarrow 0$ as $t \rightarrow 0$, (iii) and (iv) of Proposition 2.1, we conclude that 


$$
\begin{aligned}
& \int_{0}^{u^{-1}(|f(x)|)} \frac{1}{\lambda^{q_{1}}} d \Psi(\lambda) \\
= & \frac{\Psi\left(u^{-1}(|f(x)|)\right)}{\left[u^{-1}(|f(x)|)\right]^{q_{1}}}+q_{1} \int_{0}^{u^{-1}(|f(x)|)} \frac{\Psi(\lambda)}{\lambda^{q_{1}+1}} d \lambda \\
\leq & \frac{\Psi\left(u^{-1}(|f(x)|)\right)}{\left[u^{-1}(|f(x)|)\right]^{q_{1}}}+q_{1} \int_{0}^{u^{-1}(|f(x)|)} \frac{\Psi\left(u^{-1}(|f(x)|)\right)}{\lambda^{q_{1}+1}}\left[\frac{\lambda}{u^{-1}(|f(x)|)}\right]^{a_{\Psi}} d \lambda \\
= & \frac{a_{\Psi}}{a_{\Psi}-q_{1}} \frac{\Psi\left(u^{-1}(|f(x)|)\right)}{\left[u^{-1}(|f(x)|)\right]^{q_{1}}} \lesssim \frac{\Phi(|f(x)|)}{\left[u^{-1}(|f(x)|)\right]^{q_{1}}} \\
\lesssim & \frac{\Phi(|f(x)|)}{|f(x)|^{q_{1}}}[\Phi(|f(x)|)]^{q_{1} \alpha} \sim \frac{[\Phi(|f(x)|)]^{q_{1} / p_{1}}}{|f(x)|^{q_{1}}},
\end{aligned}
$$

where the second and the third inequalities to the last one depend on the facts that for any $t \in(0, \infty), \Psi\left(\Psi^{-1}(t)\right) \leq t, \Psi^{-1}(t)=\Phi^{-1}(t) t^{-\alpha}$ and $\Phi^{-1}(\Phi(t)) \geq t$. Combining (2.1) and (2.2), we conclude that

$$
\mathrm{I} \lesssim\left[\int_{\mathcal{X}} \Phi(|f(x)|) d \mu(x)\right]^{q_{1} / p_{1}} .
$$

By a method similar to the estimate for I, we also see that

$$
\mathrm{II} \lesssim\left[\int_{\mathcal{X}} \Phi(|f(x)|) d \mu(x)\right]^{q_{2} / p_{2}} .
$$

Combining the estimates for I and II, we further conclude that

$$
\int_{\mathcal{X}} \Psi(|T f(x)|) d \mu(x) \lesssim\left[\int_{\mathcal{X}} \Phi(|f(x)|) d \mu(x)\right]^{q_{1} / p_{1}}+\left[\int_{\mathcal{X}} \Phi(|f(x)|) d \mu(x)\right]^{q_{2} / p_{2}} .
$$

By a standard argument, we then know that $T$ is bounded from $L^{\Phi}(\mu)$ into $L^{\Psi}(\mu)$, which completes the proof of Theorem 2.2.

In Theorem 2.2, if we take $\alpha=0$, we then immediately obtain the following conclusion. We omit the details.

Corollary 2.3. Let $T$ be a sublinear operator of weak type $(p, p)$ for any $p \in$ $(1, \infty)$. Then $T$ is bounded on $L^{\Phi}(\mu)$, where $\Phi$ is a convex Orlicz function on $[0, \infty)$ satisfying that

$$
1<a_{\Phi} \leq b_{\Phi}<\infty .
$$

\section{Proof of Theorem 1.9}

In this section, we show Theorem 1.9. We begin with recalling some useful properties of $\delta$ in Definition 1.5 (see, for example, [9, Lemmas 5.1 and 5.2] and [12, Lemma 2.2]). 
Lemma 3.1. (i) For all balls $B \subset R \subset S, \delta(B, R) \leq \delta(B, S)$.

(ii) For any $\rho \in[1, \infty)$, there exists a positive constant $C(\rho)$, depending on $\rho$, such that, for all balls $B \subset S$ with $r_{S} \leq \rho r_{B}, \delta(B, S) \leq C(\rho)$.

(iii) For any $\alpha \in(1, \infty)$, there exists a positive constant $C(\alpha)$, depending on $\alpha$, such that, for all balls $B, \delta\left(B, \widetilde{B}^{\alpha}\right) \leq C(\alpha)$.

(iv) There exists a positive constant $c$ such that, for all balls $B \subset R \subset S$,

$$
\delta(B, S) \leq \delta(B, R)+c \delta(R, S) .
$$

In particular, if $B$ and $R$ are concentric, then $c=1$.

(v) There exists a positive constant $\widetilde{c}$ such that, for all balls $B \subset R \subset S$,

$$
\delta(R, S) \leq \widetilde{c} \delta(B, S)
$$

moreover, if $B$ and $R$ are concentric, then $\delta(R, S) \leq \delta(B, S)$.

Now we recall the following equivalent characterizations of $\operatorname{RBMO}(\mu)$ established in [12, Proposition 2.10].

Lemma 3.2. Let $\rho \in(1, \infty)$ and $f \in L_{\mathrm{loc}}^{1}(\mu)$. The following statements are equivalent:

(a) $f \in \operatorname{RBMO}(\mu)$;

(b) there exists a positive constant $C$ such that, for all balls $B$,

$$
\frac{1}{\mu(\rho B)} \int_{B}\left|f(x)-m_{\widetilde{B}} f\right| d \mu(x) \leq C
$$

and, for all doubling balls $B \subset S$,

$$
\left|m_{B}(f)-m_{S}(f)\right| \leq C \delta(B, S) .
$$

Moreover, let $\|f\|_{*}$ be the infimum of all admissible constants $C$ in (b). Then there exists a constant $\widetilde{C} \in[1, \infty)$ such that, for all $f \in \operatorname{RBMO}(\mu),\|f\|_{*} / \widetilde{C} \leq\|f\|_{\operatorname{RBMO}(\mu)} \leq$ $\widetilde{C}\|f\|_{*}$.

Corollary 3.3. There exists a positive constant $C$ such that, for all balls $B, \rho \in$ $(1, \infty), r \in[1, \infty)$ and $f \in \operatorname{RBMO}(\mu)$,

$$
\left\{\frac{1}{\mu(\rho B)} \int_{B}\left|f(x)-m_{\widetilde{B}} f\right|^{r} d \mu(x)\right\}^{1 / r} \leq C\|f\|_{\operatorname{RBMO}(\mu)} .
$$

Moreover, the infimum of all positive constants $C$ satisfying both (3.1) and (3.2) is an equivalent $\operatorname{RBMO}(\mu)$ norm of $f$. 
Proof. Let $r \in[1, \infty)$ and $f \in \operatorname{RBMO}(\mu)$. By Minkowski's inequality, [9, Corollary 6.3], Hölder's inequality, Lemmas 3.2 and 3.1(iii), we know that

$$
\begin{aligned}
& \left\{\frac{1}{\mu(\rho B)} \int_{B}\left|f(x)-m_{\widetilde{B}} f\right|^{r} d \mu(x)\right\}^{1 / r} \\
\lesssim & \left\{\frac{1}{\mu(\rho B)} \int_{B}\left|f(x)-f_{B}\right|^{r} d \mu(x)\right\}^{1 / r} \\
& +\left[\frac{\mu(B)}{\mu(\rho B)}\right]^{1 / r}\left|f_{B}-m_{B} f\right|+\left|m_{B} f-m_{\widetilde{B}} f\right| \\
\lesssim & \|f\|_{\operatorname{RBMO}(\mu)}+\left\{\frac{1}{\mu(\rho B)} \int_{B}\left|f(x)-f_{B}\right|^{r} d \mu(x)\right\}^{1 / r} \\
& +\left|m_{B} f-m_{\widetilde{B}} f\right| \lesssim\|f\|_{\operatorname{RBMO}(\mu)},
\end{aligned}
$$

which, together with Hölder's inequality, then completes the proof of Corollary 3.3.

We also need to recall some known conclusions from [2, Sections 4.1 and 7.1] and [9, Corollary 3.6].

Lemma 3.4. Let $p \in(1, \infty)$.

(i) Let $r \in(1, p)$ and $\rho \in(0, \infty)$. The following maximal operators, defined by setting, for all $f \in L^{p}(\mu)$ and $x \in \mathcal{X}$,

$$
\begin{aligned}
& M_{r, \rho} f(x):=\sup _{Q \ni x}\left[\frac{1}{\mu(\rho Q)} \int_{Q}|f(x)|^{r} d \mu(x)\right]^{\frac{1}{r}}, \\
& N f(x):=\sup _{Q \ni x, Q \text { doubling }} \frac{1}{\mu(Q)} \int_{Q}|f(x)| d \mu(x)
\end{aligned}
$$

and

$$
M_{(\rho)} f(x):=\sup _{Q \ni x} \frac{1}{\mu(\rho Q)} \int_{Q}|f(x)| d \mu(x),
$$

are bounded on $L^{p}(\mu)$.

(ii) The Calderon-Zygmund operator $T$ as in (1.10) is bounded on $L^{p}(\mu)$ if $T$ is bounded on $L^{2}(\mu)$;

(iii) $|f(x)| \leq N f(x)$ for almost every $x \in \mathcal{X}$.

Recall that the sharp maximal operator $M^{\#}$ in [2] is defined by setting, for all $f \in L_{\mathrm{loc}}^{1}(\mu)$ and $x \in \mathcal{X}$,

$$
M^{\#} f(x):=\sup _{B \ni x} \frac{1}{\mu(6 B)} \int_{B}\left|f(x)-m_{\widetilde{B}} f\right| d \mu(x)+\sup _{(Q, R) \in \Delta_{x}} \frac{\left|m_{Q} f-m_{R} f\right|}{\delta(Q, R)},
$$


where $\Delta_{x}:=\{(Q, R): x \in Q \subset R$ and $Q, R$ are doubling balls $\}$, and the maximal operator $T^{*}$, associated with the Calderon-Zygmund operator $T$, is defined by setting, for all $f \in L^{\infty}(\mu)$ with bounded support and $x \in \mathcal{X}$,

$$
T^{*} f(x):=\sup _{\epsilon>0}\left|T_{\epsilon} f(x)\right|,
$$

where

$$
T_{\epsilon} f(x):=\int_{d(x y) \geq \epsilon} K(x, y) f(y) d \mu(y) .
$$

The following discrete version, $\widetilde{\delta}(B, S)$, of $\delta(B, S)$ defined in Definition 1.5, was first introduced by Bui and Duong [2] in non-homogeneous spaces, which is more close to the quantity $K_{Q, R}$ introduced by Tolsa [22] (see also [23, 24]) in the setting of non-doubling measures. For any two balls $B \subset S$, let $\widetilde{\delta}(B, S)$ be defined by

$$
\widetilde{\delta}(B, S):=1+\sum_{k=1}^{N_{B, S}} \frac{\mu\left(6^{k} B\right)}{\lambda\left(c_{B}, 6^{k} r_{B}\right)},
$$

where $N_{B, S}$ is the smallest integer satisfying $6^{N_{B, S}} r_{B} \geq r_{S}$. Then, obviously, $\delta(B, S) \lesssim \widetilde{\delta}(B, S)$. As was pointed by Bui and Duong [2], in general, it is not true that $\delta(B, S) \sim \widetilde{\delta}(B, S)$ without the assumption (1.2).

As a variant of [22, Lemma 2.1], we have the following properties of $\widetilde{\delta}(B, S)$.

Lemma 3.5. (i) For all balls $B \subset R \subset S, \widetilde{\delta}(B, R) \leq 2 \widetilde{\delta}(B, S)$.

(ii) For any $\rho \in[1, \infty)$, there exists a positive constant $C(\rho)$, depending on $\rho$, such that, for all balls $B \subset S$ with $r_{S} \leq \rho r_{B}, \widetilde{\delta}(B, S) \leq C(\rho)$.

(iii) There exists a positive constant $C$ such that, for all balls $B, \widetilde{\delta}(B, \widetilde{B}) \leq C$.

(iv) There exists a positive constant $c$ such that, for all balls $B \subset R \subset S$,

$$
\widetilde{\delta}(B, S) \leq \widetilde{\delta}(B, R)+c \widetilde{\delta}(R, S)
$$

(v) There exists a positive constant $\widetilde{c}$ such that, for all balls $B \subset R \subset S$,

$$
\widetilde{\delta}(R, S) \leq \widetilde{c} \widetilde{\delta}(B, S)
$$

Proof. The properties (i) and (ii) are obvious.

Let us prove (iii). To this end, let $N$ be the first integer such that $6^{N} B$ is doubling. For $k \in\{1, \cdots, N-1\}$, we have $\mu\left(6^{k+1} B\right)>\beta_{6} \mu\left(6^{k} B\right)$. Thus, $\mu\left(6^{k} B\right)<\frac{\mu\left(6^{N} B\right)}{\beta_{6}^{N-k}}$ for $k \in\{1, \cdots, N-1\}$. By this, together with (1.3) and the fact that $\beta_{6}>C_{\lambda}^{\log _{2} 12}$ 
(see (1.5)), we conclude that

$$
\begin{aligned}
\widetilde{\delta}\left(B, 6^{N} B\right) & =1+\sum_{k=1}^{N} \frac{\mu\left(6^{k} B\right)}{\lambda\left(c_{B}, 6^{k} r_{B}\right)} \leq 2+\sum_{k=1}^{N-1} \frac{\mu\left(6^{k} B\right)}{\lambda\left(c_{B}, 6^{k} r_{B}\right)} \\
& \lesssim 1+\sum_{k=1}^{N-1}\left(\frac{C_{\lambda}^{\log _{2} 12}}{\beta_{6}}\right)^{N-k} \frac{\mu\left(6^{N} B\right)}{\lambda\left(c_{B}, 6^{N} r_{B}\right)} \lesssim 1+\sum_{k=1}^{\infty}\left(\frac{C_{\lambda}^{\log _{2} 12}}{\beta_{6}}\right)^{k} \lesssim 1,
\end{aligned}
$$

which completes the proof of (iii).

Next we show (iv). Obviously, $N_{B, R} \leq N_{B, S}+1$. If $N_{B, R}=N_{B, S}$ or $N_{B, R}=$ $N_{B, S}+1$, there exists nothing to prove. If $N_{B, R}<N_{B, S}$, from the fact that $N_{B, S} \leq$ $N_{B, R}+N_{R, S}, 6^{N_{B, R}} r_{B} \geq r_{R}, 6^{N_{B, R}-1} B \subset 2 R$ and (1.3), it follows that

$$
\begin{aligned}
\widetilde{\delta}(B, S) & \leq \widetilde{\delta}(B, R)+\sum_{k=N_{B, R}+1}^{N_{B, S}} \frac{\mu\left(6^{k} B\right)}{\lambda\left(c_{B}, 6^{k} r_{B}\right)} \leq \widetilde{\delta}(B, R)+\sum_{k=N_{B, R}+1}^{N_{B, R}+N_{R, S}} \frac{\mu\left(6^{k} B\right)}{\lambda\left(c_{B}, 6^{k} r_{B}\right)} \\
& \leq \widetilde{\delta}(B, R)+\sum_{k=1}^{N_{R, S}} \frac{\mu\left(6^{k+N_{B, R}} B\right)}{\lambda\left(c_{B}, 6^{k+N_{B, R}} r_{B}\right)} \leq \widetilde{\delta}(B, R)+\sum_{k=1}^{N_{R, S}} \frac{\mu\left(6^{k+2} R\right)}{\lambda\left(c_{B}, 6^{k} r_{R}\right)} \\
& \leq \widetilde{\delta}(B, R)+c \sum_{k=1}^{N_{R, S}} \frac{\mu\left(6^{k+2} R\right)}{\lambda\left(c_{R}, 6^{k+2} r_{R}\right)} \leq \widetilde{\delta}(B, R)+c\left(1+\sum_{k=1}^{N_{R, S}} \frac{\mu\left(6^{k} R\right)}{\lambda\left(c_{R}, 6^{k} r_{R}\right)}\right) \\
& \leq \widetilde{\delta}(B, R)+c \widetilde{\delta}(R, S),
\end{aligned}
$$

where $c$ is a positive constant, which shows (iv).

For (v), we first prove that $N_{B, R}+N_{R, S} \leq N_{B, S}+1$. Since

$$
r_{R}=6^{-N_{R, S}+1} 6^{N_{R, S}-1} r_{R} \leq 6^{-N_{R, S}+1} r_{S} \leq 6^{-N_{R, S}+1} 6^{N_{B, S}} r_{B} \leq 6^{N_{B, S}-N_{R, S}+1} r_{B},
$$

we obtain $N_{B, R} \leq N_{B, S}-N_{R, S}+1$. From this, $6^{N_{B, R}-1} r_{B}<r_{R} \leq 6^{N_{B, R}} r_{B}$ and (1.3), it follows that

$$
\begin{aligned}
\widetilde{\delta}(R, S) & \leq 1+\sum_{k=1}^{N_{R, S}} \frac{\mu\left(6^{k+N_{B, R}} B\right)}{\lambda\left(c_{R}, 6^{k+N_{B, R}-1} r_{B}\right)} \lesssim 1+\sum_{k=N_{B, R}+1}^{N_{B, R}+N_{R, S}} \frac{\mu\left(6^{k} B\right)}{\lambda\left(c_{R}, 6^{k-1} r_{B}\right)} \\
& \lesssim 1+\sum_{k=N_{B, R}+1}^{N_{B, S}+1} \frac{\mu\left(6^{k} B\right)}{\lambda\left(c_{B}, 6^{k-1} r_{B}\right)} \lesssim 1+\sum_{k=1}^{N_{B, S}} \frac{\mu\left(6^{k} B\right)}{\lambda\left(c_{B}, 6^{k} r_{B}\right)} \sim \widetilde{\delta}(B, S),
\end{aligned}
$$

which completes the proof of (v) and hence Lemma 3.5.

Now we introduce the maximal operator $\widetilde{M}^{\#}$ associated with $\widetilde{\delta}$. 
Definition 3.6. For all $f \in L_{\mathrm{loc}}^{1}(\mu)$ and $x \in \mathcal{X}$, let

$$
\widetilde{M}^{\#} f(x):=\sup _{B \ni x} \frac{1}{\mu(6 B)} \int_{B}\left|f(x)-m_{\widetilde{B}} f\right| d \mu(x)+\sup _{(Q, R) \in \Delta_{x}} \frac{\left|m_{Q} f-m_{R} f\right|}{\widetilde{\delta}(Q, R)},
$$

where $\Delta_{x}:=\{(Q, R): \quad x \in Q \subset R$ and $Q, R$ are doubling balls $\}$.

Remark 3.7. The sharp maximal operator $\widetilde{M}^{\#}$ has the following useful properties.

(i) By the fact that, for all doubling balls $Q \subset R, \delta(Q, R) \lesssim \widetilde{\delta}(Q, R)$, we easily see that $\widetilde{M}^{\#} f(x) \lesssim M^{\#} f(x)$ for all $x \in \mathcal{X}$.

(ii) From (i), together with the corresponding properties of $M^{\#}$ proved by Bui and Duong in [2, Section 4.1], we infer that $\widetilde{M}^{\#}$ is of weak type $(1,1)$ and bounded on $L^{p}(\mu)$ for all $p \in(1, \infty)$.

(iii) By an argument similar to that used in the proof of [22, Remark 6.1], we conclude that $\widetilde{M}^{\#}|f|(x) \leq 5 \beta_{6} \widetilde{M}^{\#} f(x)$ for all $x \in \mathcal{X}$. We omit the details.

The following theorem is a variant of [2, Theorem 4.2].

Theorem 3.8. Let $f \in L_{\text {loc }}^{1}(\mu)$ satisfying that $\int_{\mathcal{X}} f(x) d \mu(x)=0$ when $\|\mu\|:=$ $\mu(\mathcal{X})<\infty$. Assume that, for some $p \in(1, \infty)$, $\inf \{1, N f\} \in L^{p}(\mu)$. Then there exists a positive constant $C$, independent of $f$, such that

$$
\|N f\|_{L^{p}(\mu)} \leq C\left\|\widetilde{M}^{\#} f\right\|_{L^{p}(\mu)} .
$$

Proof. By Lemma 3.5 and Remark 3.7, repeating the argument used in the proof of [2, Theorem 4.2], we obtain the desired conclusion. We omit the details, which completes the proof of Theorem 3.8.

Remark 3.9. We observe that Theorem 3.8 improves [2, Theorem 4.2] by Remark 3.7(i).

We now establish the $L^{p}(\mu)$-boundedness, for all $p \in(1, \infty)$, of commutators of Calderón-Zygmund operators with $\operatorname{RBMO}(\mu)$ functions without the assumption (1.2), which essentially improves [2, Theorem 7.6].

Theorem 3.10. Let $b \in \mathrm{RBMO}(\mu)$ and T be a Calderón-Zygmund operator which is bounded on $L^{2}(\mu)$. Then the commutator $[b, T]$ as in (1.13) is bounded on $L^{p}(\mu)$ for all $p \in(1, \infty)$.

To prove Theorem 3.10, we need some technical lemmas adapting from [22].

Lemma 3.11. Let $f \in \operatorname{RBMO}(\mu), q \in(0, \infty)$ and, for all $x \in \mathcal{X}$,

$$
f_{q}(x):=\left\{\begin{array}{cl}
f(x), & \text { if }|f(x)| \leq q, \\
q \frac{f(x)}{\mid f(x)}, & \text { if }|f(x)|>q .
\end{array}\right.
$$

Then $f_{q} \in \operatorname{RBMO}(\mu)$ and there exists a positive constant $C$, independent of $f$, such that $\left\|f_{q}\right\|_{\mathrm{RBMO}(\mu)} \leq C\|f\|_{\mathrm{RBMO}(\mu)}$. 
Proof. It is obvious that $\||f|\|_{\mathrm{RBMO}(\mu)} \lesssim\|f\|_{\mathrm{RBMO}(\mu)}$, which further leads to

$$
\|\max \{f, g\}\| \lesssim\|f\|_{\mathrm{RBMO}(\mu)}+\|g\|_{\mathrm{RBMO}(\mu)}
$$

and

$$
\|\min \{f, g\}\| \lesssim\|f\|_{\operatorname{RBMO}(\mu)}+\|g\|_{\operatorname{RBMO}(\mu)} .
$$

Then, by an argument similar to that used in the proof of [22, Lemma 3.3], we obtain the desired result, which completes the proof of Lemma 3.11.

The following two lemmas are completely analogous to [22, Lemma 9.2] and [22, Lemma 9.3]. We omit the details.

Lemma 3.12. There exists some positive constant $P$ (big enough), depending only on $C_{\lambda}$ in (1.3), such that, if $m \in \mathbb{N}, B_{1} \subset B_{2} \subset \cdots \subset B_{m}$ are concentric balls with $\widetilde{\delta}\left(B_{i}, B_{i+1}\right)>P$ for $i \in\{1, \cdots, m-1\}$, then there exists a positive constant $C$, depending only on $C_{\lambda}$, such that

$$
\sum_{i=1}^{m-1} \widetilde{\delta}\left(B_{i}, B_{i+1}\right) \leq C \widetilde{\delta}\left(B_{1}, B_{m}\right) .
$$

Lemma 3.13. There exists a positive constant $P_{0}$ (big enough), depending on $C_{\lambda}$ in (1.3) and $\beta_{6}$ as in (1.5) with $\alpha=6$, such that, if $x \in \mathcal{X}$ is some fixed point and $\left\{f_{B}\right\}_{B \ni x}$ is a collection of numbers such that $\left|f_{B}-f_{S}\right| \leq C_{x}$ for all doubling balls $B \subset S$ with $x \in B$ such that $\widetilde{\delta}(B, S) \leq P_{0}$, then there exists a positive constant $C$, depending only on $C_{\lambda}, \beta_{6}$ and $P_{0}$, such that

$$
\left|f_{B}-f_{S}\right| \leq C \widetilde{\delta}(B, S) C_{x} \quad \text { for all doubling balls } B \subset S \text { with } x \in B .
$$

Now we sketch the proof of Theorem 3.10 and refer to [2, Theorem 7.6] for more details.

Proof of Theorem 3.10. To show Theorem 3.10, it suffices to show that, for all $f \in L^{p}(\mu)$ with $p \in(1, \infty)$ and $x \in \mathcal{X}$,

$$
\widetilde{M}^{\#}([b, T] f)(x) \lesssim\|b\|_{\operatorname{RBMO}(\mu)}\left[M_{r, 5} f(x)+M_{r, 6}(T f)(x)+T_{*} f(x)\right] .
$$

We assume (3.4) for a moment and then show that $[b, T]$ is bounded on $L^{p}(\mu)$ for all $p \in(1, \infty)$. Indeed, under the assumption that $T$ is bounded on $L^{2}(\mu)$, by Cotlar's inequality ([2, Theorem 6.6]), (i) and (ii) of Lemma 3.4, we conclude that $T_{*}$ is bounded on $L^{p}(\mu)$ for all $p \in(1, \infty)$. This fact, together with (3.4), (i) and (ii) of Lemma 3.4, implies that $\widetilde{M}^{\#}([b, T])$ is bounded on $L^{p}(\mu)$ for all $p \in(1, \infty)$. By Lemma 3.11 and a standard limit argument, without loss of generality, we may assume that $b$ is a bounded function, which, together with (i) and (ii) of Lemma 3.4, implies that $\inf \{1, N([b, T] f)\} \in L^{p}(\mu)$ if $f \in L^{p}(\mu)$. We now consider two cases for $\|\mu\|$. 
Case (i) $\|\mu\|=\infty$. In this case, applying Lemma 3.4(iii) and Theorem 3.8, we know that $[b, T]$ is bounded on $L^{p}(\mu)$ for all $p \in(1, \infty)$.

Case (ii) $\|\mu\|<\infty$. In this case, by Corollary 3.3 and the Lebesgue dominated convergence theorem, we see that, for all $r \in(1, \infty)$,

$$
\left[\frac{1}{\mu(\mathcal{X})} \int_{\mathcal{X}}\left|b(x)-b_{\mathcal{X}}\right|^{r} d \mu(x)\right]^{1 / r} \lesssim\|b\|_{\operatorname{RBMO}(\mu)}
$$

where $b_{\mathcal{X}}:=\frac{1}{\mu(\mathcal{X})} \int_{\mathcal{X}} b(y) d \mu(y)$. Write

$$
N([b, T] f) \leq N\left([b, T] f-m_{\mathcal{X}}([b, T] f)\right)+\left|m_{\mathcal{X}}([b, T] f)\right| .
$$

Notice that $\int_{\mathcal{X}}\left\{[b, T] f(x)-m_{\mathcal{X}}([b, T] f)\right\} d \mu(x)=0$. Then by Theorem 3.8, the fact that $\widetilde{M}^{\#}\left([b, T] f-m_{\mathcal{X}}([b, T] f)=\widetilde{M}^{\#}([b, T] f)\right.$ and the $L^{p}(\mu)$-boundedness of $\widetilde{M}^{\#}([b, T])$ for all $p \in(1, \infty)$, we see that

$$
\begin{aligned}
\left\|N\left([b, T] f-m_{\mathcal{X}}([b, T] f)\right)\right\|_{L^{p}(\mu)} & \lesssim\left\|\widetilde{M}^{\#}\left([b, T] f-m_{\mathcal{X}}([b, T] f)\right)\right\|_{L^{p}(\mu)} \\
& \sim\left\|\widetilde{M}^{\#}([b, T] f)\right\|_{L^{p}(\mu)} \lesssim\|f\|_{L^{p}(\mu)} .
\end{aligned}
$$

For the term $\left|m_{\mathcal{X}}([b, T] f)\right|$, we further write $|[b, T] f| \leq\left|\left(b-b_{\mathcal{X}}\right) T f\right|+\left|T\left(\left(b-b_{\mathcal{X}}\right) f\right)\right|$, which, together with Hölder's inequality, (3.5) and the $L^{q}(\mu)$-boundedness of $T$ for all $q \in(1, p]$, further implies that

$$
\left\|m_{\mathcal{X}}([b, T] f)\right\|_{L^{p}(\mu)} \lesssim\|f\|_{L^{p}(\mu)} .
$$

Thus, $[b, T]$ is also bounded on $L^{p}(\mu)$ for all $p \in(1, \infty)$ in this case.

Now we prove (3.4). By $b \in \operatorname{RBMO}(\mu)$ and Definition 1.7, there exists a family of numbers, $\left\{b_{B}\right\}_{B}$, satisfying that, for all balls $B$,

$$
\int_{B}\left|b(x)-b_{B}\right| d \mu(x) \leq 2 \mu(6 B)\|b\|_{\operatorname{RBMO}(\mu)}
$$

and, for all balls $Q \subset R$,

$$
\left|b_{Q}-b_{R}\right| \leq 2 \delta(Q, R)\|b\|_{\operatorname{RBMO}(\mu)}
$$

For all balls $Q$, let

$$
h_{Q}:=m_{Q}\left(T\left(\left(b-b_{Q}\right) f \chi_{\mathcal{X} \backslash(6 / 5) Q}\right)\right) .
$$

Next we show that, for all $x \in \mathcal{X}$ and balls $B$ with $B \ni x$,

$$
\frac{1}{\mu(6 B)} \int_{B}\left|[b, T] f(y)-h_{B}\right| d \mu(y) \lesssim\|b\|_{\mathrm{RBMO}(\mu)}\left[M_{r, 5} f(x)+M_{r, 6}(T f)(x)\right]
$$


and, for all $x \in Q \subset R$,

$$
\left|h_{Q}-h_{R}\right| \lesssim\|b\|_{\operatorname{RBMO}(\mu)}\left[M_{r, 5} f(x)+T_{*} f(x)\right] \delta(Q, R) \widetilde{\delta}(Q, R) .
$$

The proof of (3.6) is analogous to that of [22, (9.5)] with a slight modification, and we omit the details.

To prove (3.7), for two balls $Q \subset R$, let $N:=1+N_{Q, R}$. Then, we control $\left|h_{Q}-h_{R}\right|$ by the following five terms:

$$
\begin{aligned}
\left|h_{Q}-h_{R}\right| \leq & \left|m_{Q}\left(T\left(\left(b-b_{Q}\right) f \chi_{6 Q \backslash(6 / 5) Q}\right)\right)\right|+\left|m_{Q}\left(T\left(\left(b_{Q}-b_{R}\right) f \chi_{\mathcal{X} \backslash 6 Q}\right)\right)\right| \\
& +\left|m_{Q}\left(T\left(\left(b-b_{R}\right) f \chi_{6^{N} Q \backslash 6 Q}\right)\right)\right|+\mid m_{Q}\left(T\left(\left(b-b_{R}\right) f \chi_{\mathcal{X} \backslash 6^{N} Q}\right)\right) \\
& -m_{R}\left(T\left(\left(b-b_{R}\right) f \chi_{\mathcal{X} \backslash 6^{N} Q}\right)\right)|+| m_{R}\left(T\left(\left(b-b_{R}\right) f \chi_{6^{N} Q \backslash(6 / 5) R}\right)\right) \mid \\
= & : \mathrm{M}_{1}+\mathrm{M}_{2}+\mathrm{M}_{3}+\mathrm{M}_{4}+\mathrm{M}_{5} .
\end{aligned}
$$

By a slight modified argument similar to that used in the proof of [2, Theorem 7.6], we conclude that, for all $x \in \mathcal{X}$,

$$
\begin{aligned}
\mathrm{M}_{1}+\mathrm{M}_{4}+\mathrm{M}_{5} & \lesssim\|b\|_{\mathrm{RBMO}(\mu)} M_{r, 5} f(x) \\
\mathrm{M}_{2} & \lesssim\|b\|_{\operatorname{RBMO}(\mu)} \delta(Q, R)\left[T_{*} f(x)+M_{r, 5} f(x)\right]
\end{aligned}
$$

and

$$
\mathrm{M}_{3} \lesssim\|b\|_{\mathrm{RBMO}(\mu)} \delta(Q, R) \widetilde{\delta}(Q, R) M_{r, 5} f(x),
$$

which further implies (3.7).

By an argument similar to the proof of [22, Theorem 9.1], together with Lemmas 3.12 and 3.13, $\delta(Q, R) \lesssim \widetilde{\delta}(Q, R)$, (3.6) and (3.7), we obtain (3.4), which completes the proof of Theorem 3.10.

To prove Theorem 1.9, we need to recall some notation from [7]. For $k \in \mathbb{N}$ and $i \in\{1, \cdots, k\}$, the family of all finite subsets $\sigma:=\{\sigma(1), \cdots, \sigma(i)\}$ of $\{1, \cdots, k\}$ with $i$ different elements is denoted by $C_{i}^{k}$. For any $\sigma \in C_{i}^{k}$, the complementary sequence $\sigma^{\prime}$ is given by $\sigma^{\prime}:=\{1, \cdots, k\} \backslash \sigma$. For any $\sigma:=\{\sigma(1), \cdots, \sigma(i)\} \in C_{i}^{k}$ and $k$-tuple $r:=\left(r_{1}, \cdots, r_{k}\right)$, we write that $1 / r_{\sigma}:=1 / r_{\sigma(1)}+\cdots+1 / r_{\sigma(i)}$ and $1 / r_{\sigma^{\prime}}:=$ $1 / r-1 / r_{\sigma}$, where $1 / r:=1 / r_{1}+\cdots+1 / r_{k}$. Let $\vec{b}:=\left(b_{1}, \cdots, b_{k}\right)$ be a finite family of locally integrable functions. For all $i \in\{1, \cdots, k\}$ and $\sigma:=\{\sigma(1), \cdots, \sigma(i)\} \in C_{i}^{k}$, we let $b_{\sigma}:=b_{\sigma(1)} \cdots b_{\sigma(i)}, \vec{b}_{\sigma}:=\left(b_{\sigma(1)}, \cdots, b_{\sigma(i)}\right)$,

$$
\left\|\vec{b}_{\sigma}\right\|_{\operatorname{RBMO}(\mu)}:=\left\|b_{\sigma(1)}\right\|_{\operatorname{RBMO}(\mu)} \cdots\left\|b_{\sigma(i)}\right\|_{\operatorname{RBMO}(\mu)}
$$

and, for any $y, z \in \mathcal{X}$ and any ball $B$ in $\mathcal{X}$,

$$
\left[m_{\widetilde{B}}(b)-b(z)\right]_{\sigma}:=\left[m_{\widetilde{B}}\left(b_{\sigma(1)}\right)-b_{\sigma(1)}(z)\right] \cdots\left[m_{\widetilde{B}}\left(b_{\sigma(i)}\right)-b_{\sigma(i)}(z)\right] .
$$


For any $\vec{b}:=\left(b_{1}, \cdots, b_{k}\right)$, we simply write

$$
\|\vec{b}\|_{\operatorname{RBMO}(\mu)}:=\left\|b_{1}\right\|_{\operatorname{RBMO}(\mu)} \cdots\left\|b_{k}\right\|_{\operatorname{RBMO}(\mu)} .
$$

For any $\sigma \in C_{i}^{k}$, we set

$$
T_{\vec{b}_{\sigma}}:=\left[b_{\sigma(i)}, \cdots,\left[b_{\sigma(1)}, T\right] \cdots\right] .
$$

In particular, when $\sigma:=\{1, \cdots, k\}, T_{\vec{b}_{\sigma}}$ coincides with $T_{\vec{b}}$ as in (1.12).

Now we turn to the proof of Theorem 1.9.

Proof of Theorem 1.9. To prove Theorem 1.9, by Corollary 2.3, it suffices to prove that $T_{\vec{b}}$ is bounded on $L^{p}(\mu)$ for all $p \in(1, \infty)$. We show this by induction on $k$.

By Theorem 3.10, the conclusion is valid for $k=1$. Now assume that $k \geq 2$ is an integer and for any $i \in\{1, \cdots, k-1\}$ and any subset $\sigma=\{\sigma(1), \cdots, \sigma(i)\}$ of $\{1, \cdots, k\}, T_{\vec{b}_{\sigma}}$ is bounded on $L^{\Phi}(\mu)$.

The case that $\|\mu\|<\infty$ can be proved by a way similar to the proof of Theorem 3.10 , and we omit the details. Thus, without loss of generality, we may assume that $\|\mu\|=\infty$. Let $p \in(1, \infty)$. We first claim that, for all $r \in(1, \infty), f \in L^{p}(\mu)$, and $x \in \mathcal{X}$,

$$
\begin{aligned}
\widetilde{M}^{\#}\left(T_{\vec{b}} f\right)(x) \lesssim & \|\vec{b}\|_{\operatorname{RBMO}(\mu)}\left[M_{r, 6}(T f)(x)+M_{r, 5} f(x)\right] \\
& +\sum_{i=1}^{k-1} \sum_{\sigma \in C_{i}^{k}}\left\|\vec{b}_{\sigma}\right\|_{\operatorname{RBMO}(\mu)} M_{r, 6}\left(T_{\vec{b}_{\sigma^{\prime}}} f\right)(x) .
\end{aligned}
$$

Once (3.8) is proved, by an argument similar to that used in the proof of Theorem 3.10 , we conclude that, for all $p \in(1, \infty)$ and $f \in L^{p}(\mu)$,

$$
\begin{aligned}
\left\|T_{\vec{b}} f\right\|_{L^{p}(\mu)} \leq & \left\|N\left(T_{\vec{b}} f\right)\right\|_{L^{p}(\mu)} \lesssim\left\|\widetilde{M}^{\#}\left(T_{\vec{b}} f\right)\right\|_{L^{p}(\mu)} \\
\lesssim & \|\vec{b}\|_{\operatorname{RBMO}(\mu)}\left[\left\|M_{r, 6}(T f)\right\|_{L^{p}(\mu)}+\left\|M_{r, 5}(f)\right\|_{L^{p}(\mu)}\right] \\
& +\sum_{i=1}^{k-1} \sum_{\sigma \in C_{i}^{k}}\left\|\vec{b}_{\sigma}\right\|_{\operatorname{RBMO}(\mu)}\left\|M_{r, 6}\left(T_{\vec{b}_{\sigma^{\prime}}} f\right)\right\|_{L^{p}(\mu)} \\
\lesssim & \|\vec{b}\|_{\operatorname{RBMO}(\mu)}\left[\|T f\|_{L^{p}(\mu)}+\|f\|_{L^{p}(\mu)}+\sum_{i=1}^{k-1} \sum_{\sigma \in C_{i}^{k}}\left\|T_{\vec{b}_{\sigma^{\prime}}} f\right\|_{L^{p}(\mu)}\right] \\
\lesssim & \|\vec{b}\|_{\operatorname{RBMO}(\mu)}\|f\|_{L^{p}(\mu)},
\end{aligned}
$$

which is desired. 
As in the proof of [22, Theorem 9.1], to prove (3.8), it suffices to show that, for all $x \in \mathcal{X}$ and balls $B$ with $B \ni x$,

$$
\begin{aligned}
\frac{1}{\mu(6 B)} \int_{B}\left|T_{\vec{b}} f(y)-h_{B}\right| d \mu(y) \lesssim & \|\vec{b}\|_{\operatorname{RBMO}(\mu)}\left[M_{r, 5} f(x)+M_{r, 6}(T f)(x)\right] \\
& +\sum_{i=1}^{k-1} \sum_{\sigma \in C_{i}^{k}}\left\|\vec{b}_{\sigma}\right\|_{\operatorname{RBMO}(\mu)} M_{r, 6}\left(T_{\vec{b}_{\sigma^{\prime}}} f\right)(x)
\end{aligned}
$$

and, for an arbitrary ball $Q$, a doubling ball $R$ with $Q \subset R$ and $x \in Q$,

$$
\begin{aligned}
\left|h_{Q}-h_{R}\right| \lesssim & {[\widetilde{\delta}(Q, R)]^{k+1}\left\{\|\vec{b}\|_{\operatorname{RBMO}(\mu)}\left[M_{r, 5} f(x)+M_{r, 6}(T f)(x)\right]\right.} \\
& \left.+\sum_{i=1}^{k-1} \sum_{\sigma \in C_{i}^{k}}\left\|\vec{b}_{\sigma}\right\|_{\operatorname{RBMO}(\mu)} M_{r, 6}\left(T_{\vec{b}_{\sigma^{\prime}}} f\right)(x)\right\}
\end{aligned}
$$

where

$$
h_{Q}:=m_{Q}\left(T\left(\left[\left(m_{\widetilde{Q}}\left(b_{1}\right)-b_{1}\right) \cdots\left(m_{\widetilde{Q}}\left(b_{k}\right)-b_{k}\right)\right] f \chi_{\mathcal{X} \backslash \frac{6}{5} Q}\right)\right)
$$

and

$$
h_{R}:=m_{R}\left(T\left(\left[\left(m_{R}\left(b_{1}\right)-b_{1}\right) \cdots\left(m_{R}\left(b_{k}\right)-b_{k}\right)\right] f \chi_{\mathcal{X} \backslash \frac{6}{5} R}\right)\right) .
$$

Let us first prove (3.9). With the aid of the formula that, for all $y, z \in \mathcal{X}$,

$$
\prod_{i=1}^{k}\left[m_{\widetilde{Q}}\left(b_{i}\right)-b_{i}(z)\right]=\sum_{i=0}^{k} \sum_{\sigma \in C_{i}^{k}}[b(y)-b(z)]_{\sigma^{\prime}}\left[m_{\widetilde{Q}}(b)-b(y)\right]_{\sigma}
$$

where, if $i=0$, we set $\sigma^{\prime}=\{1, \cdots, k\}, \sigma=\emptyset$ and $\left[m_{\widetilde{Q}}(b)-b(y)\right]_{\emptyset}=1$, it is easy to prove that, for all $y \in \mathcal{X}$,

$$
T_{\vec{b}} f(y)=T\left(\prod_{i=1}^{k}\left[m_{\widetilde{Q}}\left(b_{i}\right)-b_{i}\right] f\right)(y)-\sum_{i=1}^{k} \sum_{\sigma \in C_{i}^{k}}\left[m_{\widetilde{Q}}(b)-b(y)\right]_{\sigma} T_{\vec{b}_{\sigma^{\prime}}} f(y),
$$

where, if $i=k, T_{\vec{b}_{\sigma^{\prime}}} f:=T f$. Therefore, for all balls $Q \ni x$,

$$
\begin{aligned}
& \frac{1}{\mu(6 Q)} \int_{Q}\left|T_{\vec{b}} f(y)-h_{Q}\right| d \mu(y) \\
\leq & \frac{1}{\mu(6 Q)} \int_{Q}\left|T\left(\prod_{i=1}^{k}\left[m_{\widetilde{Q}}\left(b_{i}\right)-b_{i}\right] f \chi_{\frac{6}{5} Q}\right)(y)\right| d \mu(y)
\end{aligned}
$$




$$
\begin{aligned}
& +\sum_{i=1}^{k} \sum_{\sigma \in C_{i}^{k}} \frac{1}{\mu(6 Q)} \int_{Q}\left|\left[m_{\widetilde{Q}}(b)-b(y)\right]_{\sigma}\right|\left|T_{\vec{b}_{\sigma^{\prime}}} f(y)\right| d \mu(y) \\
& +\frac{1}{\mu(6 Q)} \int_{Q}\left|T\left(\prod_{i=1}^{k}\left[m_{\widetilde{Q}}\left(b_{i}\right)-b_{i}\right] f \chi_{\mathcal{X} \backslash \frac{6}{5} Q}\right)(y)-h_{Q}\right| d \mu(y)=: \mathrm{I}_{1}+\mathrm{I}_{2}+\mathrm{I}_{3} .
\end{aligned}
$$

From Hölder's inequality and Corollary 3.3, it follows that, for all $q \in(1, \infty)$,

$$
\int_{\frac{6}{5} Q} \prod_{i=1}^{k}\left|b_{i}(y)-m_{\widetilde{Q}}\left(b_{i}\right)\right|^{q} d \mu(y) \leq C\|\vec{b}\|_{\operatorname{RBMO}(\mu)}^{q} \mu(6 Q) .
$$

Take $s=\sqrt{r}$ and write

$$
b_{i}(y)-m_{\widetilde{Q}}\left(b_{i}\right)=b_{i}(y)-m_{\widetilde{\frac{6}{5} Q}}\left(b_{i}\right)+m_{\widetilde{\frac{6}{5} Q}}\left(b_{i}\right)-m_{\widetilde{Q}}\left(b_{i}\right)
$$

for $i \in\{1, \cdots, k\}$. By Hölder's inequality and the $L^{s}(\mu)$-boundedness of $T$ for $s \in(1, \infty),(3.12)$, we conclude that, for all $x \in Q$,

$$
\begin{aligned}
\mathrm{I}_{1} & \leq \frac{[\mu(Q)]^{\frac{1}{s^{\prime}}}}{\mu(6 Q)}\left\|T\left(\prod_{i=1}^{k}\left[m_{\widetilde{Q}}\left(b_{i}\right)-b_{i}\right] f \chi_{\frac{6}{5} Q}\right)\right\|_{L^{s}(\mu)} \\
& \lesssim \frac{[\mu(Q)]^{1-\frac{1}{s}}}{\mu(6 Q)}\left\|\prod_{i=1}^{k}\left[m_{\widetilde{Q}}\left(b_{i}\right)-b_{i}\right] f \chi_{\frac{6}{5} Q}\right\|_{L^{s}(\mu)} \\
& \lesssim \frac{1}{[\mu(6 Q)]^{\frac{1}{s}}}\left\{\int_{\frac{6}{5} Q} \prod_{i=1}^{k}\left|b_{i}(y)-m_{\widetilde{Q}}\left(b_{i}\right)\right|^{s s^{\prime}} d \mu(y)\right\}^{\frac{1}{s s^{\prime}}}\left\{\int_{\frac{6}{5} Q}|f(y)|^{r} d \mu(y)\right\}^{\frac{1}{r}} \\
& \lesssim\|\vec{b}\|_{\operatorname{RBMO}(\mu)} M_{r, 5} f(x) .
\end{aligned}
$$

For $\mathrm{I}_{2}$, by (3.12), we see that, for all $x \in Q$,

$$
\begin{aligned}
\mathrm{I}_{2} \leq & \sum_{i=1}^{k} \sum_{\sigma \in C_{i}^{k}}\left\{\frac{1}{\mu(6 Q)} \int_{Q}\left|\left[b(y)-m_{\widetilde{Q}}(b)\right]_{\sigma}\right|^{r^{\prime}} d \mu(y)\right\}^{\frac{1}{r^{\prime}}} \\
& \times\left\{\frac{1}{\mu(6 Q)} \int_{Q}\left|T_{\vec{b}_{\sigma^{\prime}}} f(y)\right|^{r} d \mu(y)\right\}^{\frac{1}{r}} \lesssim \sum_{i=1}^{k} \sum_{\sigma \in C_{i}^{k}}\left\|\vec{b}_{\sigma}\right\|_{\operatorname{RBMO}(\mu)} M_{r, 6}\left(T_{\vec{b}_{\sigma^{\prime}}} f\right)(x) .
\end{aligned}
$$

To estimate $I_{3}$, we need to calculate the difference

$$
\left|T\left(\prod_{i=1}^{k}\left[m_{\widetilde{Q}}\left(b_{i}\right)-b_{i}\right] f \chi_{\mathcal{X} \backslash \frac{6}{5} Q}\right)(y)-h_{Q}\right|
$$


for all $y \in Q$. By (1.9), (1.3), (3.11), (3.12), Lemmas 3.1 and 3.2, Hölder's inequality and Corollary 3.3, we see that, for $y, y_{1}, x \in Q$,

$$
\begin{aligned}
& \left|T\left(\prod_{i=1}^{k}\left[m_{\widetilde{Q}}\left(b_{i}\right)-b_{i}\right] f \chi_{\mathcal{X} \backslash \frac{6}{5} Q}\right)(y)-T\left(\prod_{i=1}^{k}\left[m_{\widetilde{Q}}\left(b_{i}\right)-b_{i}\right] f \chi_{\mathcal{X} \backslash \frac{6}{5} Q}\right)\left(y_{1}\right)\right| \\
& \lesssim \int_{\mathcal{X} \backslash \frac{6}{5} Q} \frac{d\left(y, y_{1}\right)^{\delta}}{d(y, z)^{\delta} \lambda(y, d(y, z))} \prod_{i=1}^{k}\left|b_{i}(z)-m_{\widetilde{Q}}\left(b_{i}\right)\right||f(z)| d \mu(z) \\
& \lesssim \sum_{j=1}^{\infty} \int_{2^{j} \frac{6}{5} Q \backslash 2^{j-1} \frac{6}{5} Q} 2^{-j \delta} \frac{1}{\lambda\left(y, 2^{j} 6 r_{Q}\right)} \\
& \times \prod_{i=1}^{k}\left(\left|b_{i}(z)-m \underset{2^{j \frac{6}{5} Q}}{\widetilde{C}}\left(b_{i}\right)\right|+\left|m_{2^{j \frac{6}{5} Q}}\left(b_{i}\right)-m_{\widetilde{Q}}\left(b_{i}\right)\right|\right)|f(z)| d \mu(z) \\
& \lesssim \sum_{j=1}^{\infty} \sum_{i=0}^{k} \sum_{\sigma \in C_{i}^{k}} 2^{-j \delta} j^{k-i}\left\|\vec{b}_{\sigma^{\prime}}\right\|_{\operatorname{RBMO}(\mu)} \frac{1}{\mu\left(2^{j} 6 Q\right)} \\
& \times \int_{2^{j} \frac{6}{5} Q}\left|\left[b(z)-m_{2^{j \frac{6}{5} Q}}(b)\right]_{\sigma}\right| f(z) \mid d \mu(z) \\
& \lesssim \sum_{i=0}^{k} \sum_{\sigma \in C_{i}^{k}} \sum_{j=1}^{\infty} 2^{-j \delta} j^{k-i}\left\|\vec{b}_{\sigma}\right\|_{\operatorname{RBMO}(\mu)}\left\|\vec{b}_{\sigma^{\prime}}\right\|_{\operatorname{RBMO}(\mu)} M_{r, 5} f(x) \\
& \lesssim\|\vec{b}\|_{\mathrm{RBMO}(\mu)} M_{r, 5} f(x),
\end{aligned}
$$

where, in the third to the last inequality, we have used Lemmas 3.1 and 3.2 to conclude that, for all $i \in\{1, \cdots, k\}$,

$$
\begin{aligned}
& \left|m_{2^{j \frac{6}{5} Q}}\left(b_{i}\right)-m_{\widetilde{Q}}\left(b_{i}\right)\right| \\
\lesssim & \left\|b_{i}\right\|_{\operatorname{RBMO}(\mu)} \delta\left(\widetilde{Q}, \widetilde{2^{j}} \frac{6}{5} Q\right) \\
\lesssim & \left\|b_{i}\right\|_{\operatorname{RBMO}(\mu)}\left[\delta(\widetilde{Q}, Q)+\delta\left(Q, 2^{j} \frac{6}{5} Q\right)+\delta\left(2^{j} \frac{6}{5} Q \widetilde{Q}, \widetilde{2^{j} \frac{6}{5} Q}\right)\right] \\
\lesssim & \left\|b_{i}\right\|_{\operatorname{RBMO}(\mu)} \delta\left(Q, 2^{j} \frac{6}{5} Q\right) \lesssim j\left\|b_{i}\right\|_{\operatorname{RBMO}(\mu)} .
\end{aligned}
$$

From the above estimate and the choice of $h_{Q}$, we deduce that, for all $x, y \in Q$, 


$$
\begin{aligned}
& \left|T\left(\prod_{i=1}^{k}\left[m_{\widetilde{Q}}\left(b_{i}\right)-b_{i}\right] f \chi_{\mathcal{X} \backslash \frac{6}{5} Q}\right)(y)-h_{Q}\right| \\
\leq & \frac{1}{\mu(Q)} \int_{Q} \mid T\left(\prod_{i=1}^{k}\left[m_{\widetilde{Q}}\left(b_{i}\right)-b_{i}\right] f \chi_{\mathcal{X} \backslash \frac{6}{5} Q}\right)(y) \\
& -T\left(\prod_{i=1}^{k}\left[m_{\widetilde{Q}}\left(b_{i}\right)-b_{i}\right] f \chi_{\mathcal{X} \backslash \frac{6}{5} Q}\right)\left(y_{1}\right) \mid d \mu\left(y_{1}\right) \lesssim\|\vec{b}\|_{\operatorname{RBMO}(\mu)} M_{r, 5} f(x)
\end{aligned}
$$

and hence

$$
\mathrm{I}_{3} \lesssim\|\vec{b}\|_{\mathrm{RBMO}(\mu)} M_{r, 5} f(x)
$$

for all $x \in Q$.

Combining the estimates for $\mathrm{I}_{1}, \mathrm{I}_{2}$ and $\mathrm{I}_{3}$, we then obtain (3.9).

Next we prove (3.10). Let $Q$ be an arbitrary ball and $R$ a doubling ball in $\mathcal{X}$ such that $x \in Q \subset R$. Denote $N_{Q, R}+1$ simply by $N$. Write

$$
\begin{aligned}
& \left|h_{Q}-h_{R}\right| \\
= & \left.\mid T\left(\prod_{i=1}^{k}\left[m_{\widetilde{Q}}\left(b_{i}\right)-b_{i}\right] f \chi_{\mathcal{X} \backslash \frac{6}{5} Q}\right)\right] \\
& -m_{R}\left[T\left(\prod_{i=1}^{k}\left[m_{R}\left(b_{i}\right)-b_{i}\right] f \chi_{\mathcal{X} \backslash \frac{6}{5} R}\right)\right] \mid \\
\leq & \mid m_{Q}\left[T\left(\prod_{i=1}^{k}\left[m_{\widetilde{Q}}\left(b_{i}\right)-b_{i}\right] f \chi_{\mathcal{X} \backslash 6^{N} Q}\right)\right] \\
& -m_{R}\left[T\left(\prod_{i=1}^{k}\left[m_{\widetilde{Q}}\left(b_{i}\right)-b_{i}\right] f \chi_{\mathcal{X} \backslash 6^{N} Q}\right)\right] \mid \\
& -m_{R}\left[T\left(\prod_{i=1}^{k}\left[m_{\widetilde{Q}}\left(b_{i}\right)-b_{i}\right] f\left(\prod_{i=1}^{k}\left[m_{R}\left(b_{i}\right)-b_{i}\right] f 6^{N} Q\right)\right]\right. \\
& +\left|m_{Q}\left[T\left(\prod_{i=1}^{k}\left[m_{\widetilde{Q}}\left(b_{i}\right)-b_{i}\right] f \chi_{6^{N} Q \backslash \frac{6}{5} Q}\right)\right]\right| \\
& +\left|m_{R}\left[T\left(\prod_{i=1}^{k}\left[m_{R}\left(b_{i}\right)-b_{i}\right] f \chi_{6^{N} Q \backslash \frac{6}{5} R}\right)\right]\right|=: \mathrm{L}_{1}+\mathrm{L}_{2}+\mathrm{L}_{3}+\mathrm{L}_{4} .
\end{aligned}
$$

By an estimate similar to that for $\mathrm{I}_{3}$, together with $\delta(Q, R) \lesssim \widetilde{\delta}(Q, R)$, we see that, 
for all $x \in Q$,

$$
\mathrm{L}_{1} \lesssim\|\vec{b}\|_{\mathrm{RBMO}(\mu)}[\widetilde{\delta}(Q, R)]^{k} M_{r, 5} f(x) .
$$

To estimate $\mathrm{L}_{2}$, from (3.11) and Lemma 3.2, we deduce that, for all $y \in R$,

$$
\begin{aligned}
& \left|T\left(\prod_{i=1}^{k}\left[m_{R}\left(b_{i}\right)-b_{i}\right] f \chi_{\mathcal{X} \backslash 6^{N} Q}\right)(y)-T\left(\prod_{i=1}^{k}\left[m_{\widetilde{Q}}\left(b_{i}\right)-b_{i}\right] f \chi_{\mathcal{X} \backslash 6^{N} Q}\right)(y)\right| \\
= & \mid T\left(\prod_{i=1}^{k}\left[m_{R}\left(b_{i}\right)-b_{i}\right] f \chi_{\mathcal{X} \backslash 6^{N} Q}\right)(y) \\
& -\sum_{i=0}^{k} \sum_{\sigma \in C_{i}^{k}}\left[m_{\widetilde{Q}^{\prime}}(b)-m_{R}(b)\right]_{\sigma^{\prime}} T\left(\left[m_{R}(b)-b\right]_{\sigma} f \chi_{\mathcal{X} \backslash 6^{N} Q}\right)(y) \mid \\
\lesssim & \sum_{i=0}^{k-1} \sum_{\sigma \in C_{i}^{k}}\left\|\vec{b}_{\sigma^{\prime}}\right\|_{\operatorname{RBMO}(\mu)}[\delta(Q, R)]^{k-i}\left|T\left(\left[m_{R}(b)-b\right]_{\sigma} f \chi_{\mathcal{X} \backslash 6^{N} Q}\right)(y)\right| \\
\lesssim & \sum_{i=0}^{k-1} \sum_{\sigma \in C_{i}^{k}}\left\|\vec{b}_{\sigma^{\prime}}\right\|_{\operatorname{RBMO}(\mu)}[\delta(Q, R)]^{k-i}\left\{\left|T\left(\left[m_{R}(b)-b\right]_{\sigma} f\right)(y)\right|\right. \\
& \left.+\left|T\left(\left[m_{R}(b)-b\right]_{\sigma} f \chi_{6^{N} Q}\right)(y)\right|\right\} \\
\lesssim & \sum_{i=0}^{k-1} \sum_{\sigma \in C_{i}^{k}}\left\|\vec{b}_{\sigma^{\prime}}\right\|_{\operatorname{RBMO}(\mu)}[\delta(Q, R)]^{k-i}\left\{\sum_{j=0}^{i} \sum_{\eta \in C_{j}^{i}}\left|\left[m_{R}(b)-b(y)\right]_{\eta^{\prime}}\right|\left|T_{\vec{b}_{\eta}} f(y)\right|\right. \\
& \left.+\left|T\left(\left[m_{R}(b)-b\right]_{\sigma} f \chi_{6^{N} Q \backslash \frac{6}{5} R}\right)(y)\right|+\left|T\left(\left[m_{R}(b)-b\right]_{\sigma} f \chi_{\frac{6}{5} R}\right)(y)\right|\right\} .
\end{aligned}
$$

Applying Hölder's inequality, the fact that $R$ is doubling, and Corollary 3.3, we see that, for all $x \in Q$,

$$
\begin{aligned}
& \frac{1}{\mu(R)} \int_{R}\left|\left[b(y)-m_{R}(b)\right]_{\eta^{\prime}}\right|\left|T_{\vec{b}_{\eta}} f(y)\right| d \mu(y) \\
\lesssim & \left\|\vec{b}_{\eta^{\prime}}\right\|_{\operatorname{RBMO}(\mu)} M_{r, 6}\left(T_{\vec{b}_{\eta}} f\right)(x) .
\end{aligned}
$$

Moreover, from Corollary 3.3 and (1.8), it follows that, for all $y \in R$,

$$
\begin{aligned}
& \left|T\left(\left[m_{R}(b)-b\right]_{\sigma} f \chi_{6^{N} Q \backslash \frac{6}{5} R}\right)(y)\right| \\
\leq & \int_{6^{N} Q \backslash \frac{6}{5} R}|K(y, z)|\left|\left[m_{R}(b)-b(z)\right]_{\sigma}\right||f(z)| d \mu(z) \\
\lesssim & \frac{1}{\lambda\left(x, r_{R}\right)} \int_{6^{N} Q \backslash \frac{6}{5} R}\left|\left[m_{R}(b)-b(z)\right]_{\sigma}\right||f(z)| d \mu(z),
\end{aligned}
$$


where $r_{R}$ denotes the radius of the ball $R$. By Hölder's inequality, the fact that $6^{N-2} r_{Q}<r_{R}$, (1.3) and Corollary 3.3, we further have

$$
\begin{aligned}
& \frac{1}{\lambda\left(x, r_{R}\right)} \int_{6^{N} Q \backslash \frac{6}{5} R}\left|\left[m_{R}(b)-b(z)\right]_{\sigma}\right||f(z)| d \mu(z) \\
\lesssim & {\left[\frac{1}{\lambda\left(x, r_{R}\right)} \int_{6^{N} Q \backslash \frac{6}{5} R}\left|\left[m_{R}(b)-b(z)\right]_{\sigma}\right|^{r^{\prime}} d \mu(z)\right]^{1 / r^{\prime}} } \\
& \times\left[\frac{1}{\lambda\left(x, r_{R}\right)} \int_{6^{N} Q \backslash \frac{6}{5} R}|f(z)|^{r} d \mu(z)\right]^{1 / r} \\
\lesssim & {\left[\frac{1}{\mu\left(6^{N+1} Q\right)} \int_{6^{N} Q}\left|\left[m_{R}(b)-b(z)\right]_{\sigma}\right|^{r^{\prime}} d \mu(z)\right]^{1 / r^{\prime}} } \\
& \times\left[\frac{1}{\mu\left(5 \times 6^{N} Q\right)} \int_{6^{N} Q}|f(z)|^{r} d \mu(z)\right]^{1 / r} \lesssim\left\|\vec{b}_{\sigma}\right\|_{\operatorname{RBMO}(\mu)} M_{r, 5} f(x) .
\end{aligned}
$$

Taking the mean over $y \in R$, we obtain

$$
m_{R}\left[\left|T\left(\left[m_{R}(b)-b\right]_{\sigma} f \chi_{6^{N} Q \backslash \frac{6}{5} R}\right)\right|\right] \lesssim\left\|\vec{b}_{\sigma}\right\|_{\operatorname{RBMO}(\mu)} M_{r, 5} f(x) .
$$

By an argument similar to the estimate for $\mathrm{I}_{1}$, we see that, for all $x \in Q$,

$$
m_{R}\left[\left|T\left(\left[m_{R}(b)-b\right]_{\sigma} f \chi_{\frac{6}{5} R}\right)\right|\right] \lesssim\left\|\vec{b}_{\sigma}\right\|_{\mathrm{RBMO}(\mu)} M_{r, 5} f(x) .
$$

Noticing that $1 \lesssim \delta(Q, R) \lesssim \widetilde{\delta}(Q, R)$ and combining (3.13), (3.14) and (3.15), we then conclude that, for all $x \in Q$,

$$
\mathrm{L}_{2} \lesssim[\widetilde{\delta}(Q, R)]^{k}\left\{\sum_{i=1}^{k} \sum_{\sigma \in C_{i}^{k}}\left\|\vec{b}_{\sigma^{\prime}}\right\|_{\mathrm{RBMO}(\mu)} M_{r, 6}\left(T_{\vec{b}_{\sigma}} f(x)\right)+\|\vec{b}\|_{\operatorname{RBMO}(\mu)} M_{r, 5} f(x)\right\} .
$$

Now we deal with $\mathrm{L}_{3}$. From (1.8), Hölder's inequality and Corollary 3.3, we infer that, for all $x, y \in Q$,

$$
\begin{aligned}
& \left|T\left(\prod_{i=1}^{k}\left[m_{\widetilde{Q}}\left(b_{i}\right)-b_{i}\right] f \chi_{6^{N} Q \backslash \frac{6}{5} Q}\right)(y)\right| \\
\lesssim & \sum_{j=1}^{N-1} \frac{1}{\lambda\left(y, 6^{j} r_{Q}\right)} \int_{6^{j+1} Q \backslash 6^{j} Q} \prod_{i=1}^{k}\left|b_{i}(z)-m_{\widetilde{Q}}\left(b_{i}\right)\right| f(z) \mid d \mu(z) \\
& +\frac{1}{\lambda\left(y, r_{Q}\right)} \int_{6 Q \backslash \frac{6}{5} Q} \prod_{i=1}^{k}\left|b_{i}(z)-m_{\widetilde{Q}^{(}}\left(b_{i}\right)\right||f(z)| d \mu(z)
\end{aligned}
$$




$$
\begin{aligned}
& \lesssim \sum_{j=1}^{N-1} \frac{1}{\lambda\left(y, 6^{j} r_{Q}\right)}\left\{\int_{6^{j+1} Q} \prod_{i=1}^{k}\left|b_{i}(z)-m_{\tilde{Q}}\left(b_{i}\right)\right|^{r^{\prime}} d \mu(z)\right\}^{\frac{1}{r^{\prime}}}\left\{\int_{6^{j+1} Q}|f(z)|^{r} d \mu(z)\right\}^{\frac{1}{r}} \\
& +\frac{1}{\lambda\left(y, r_{Q}\right)}\left\{\int_{6 Q \backslash \frac{6}{5} Q} \prod_{i=1}^{k}\left|b_{i}(z)-m_{\widetilde{Q}}\left(b_{i}\right)\right|^{r^{\prime}} d \mu(z)\right\}^{\frac{1}{r^{\prime}}}\left\{\int_{6 Q}|f(z)|^{r} d \mu(z)\right\}^{\frac{1}{r}} \\
& \lesssim \sum_{j=1}^{N-1} \frac{1}{\lambda\left(y, 6^{j} r_{Q}\right)}\left\{\int _ { 6 ^ { j + 1 } Q } \prod _ { i = 1 } ^ { k } \left[\left|b_{i}(z)-m_{6^{j+1} Q}\left(b_{i}\right)\right|\right.\right. \\
& \left.\left.+\left|m_{6^{j+1} Q}\left(b_{i}\right)-m_{\widetilde{Q}}\left(b_{i}\right)\right|\right]^{r^{\prime}} d \mu(z)\right\}^{\frac{1}{r^{\prime}}} \\
& \times\left\{\int_{6^{j+1} Q}|f(z)|^{r} d \mu(z)\right\}^{\frac{1}{r}}+\|\vec{b}\|_{\operatorname{RBMO}(\mu)}\left\{\frac{1}{\lambda\left(y, r_{Q}\right)} \int_{6 Q}|f(z)|^{r} d \mu(z)\right\}^{\frac{1}{r}} \\
& \lesssim\|\vec{b}\|_{\operatorname{RBMO}(\mu)}\left\{[\delta(Q, R)]^{k} \sum_{j=1}^{N-1} \frac{\mu\left(5 \times 6^{j+1} Q\right)}{\lambda\left(y, 5 \times 6^{j+1} Q\right)}\left\{\frac{1}{\mu\left(5 \times 6^{j+1} Q\right)} \int_{6^{j+1} Q}|f(z)|^{r} d \mu(z)\right\}^{\frac{1}{r}}\right. \\
& \left.+M_{r, 5} f(x)\right\}
\end{aligned}
$$

where the last inequality follows from an argument similar to the estimate of $\mathrm{I}_{2}$. Taking the mean over $y \in Q$, we see that, for all $x \in Q$,

$\mathrm{L}_{3} \lesssim\|\vec{b}\|_{\mathrm{RBMO}(\mu)}[\delta(Q, R)]^{k} \widetilde{\delta}(Q, R) M_{r, 5} f(x) \lesssim\|\vec{b}\|_{\mathrm{RBMO}(\mu)}[\widetilde{\delta}(Q, R)]^{k+1} M_{r, 5} f(x)$.

Finally, we estimate $\mathrm{L}_{4}$. By (1.8), Hölder's inequality and Corollary 3.3, we see that, for all $y \in R$ and $x \in Q \subset R$,

$$
\begin{aligned}
&\left|T\left(\prod_{i=1}^{k}\left[m_{R}\left(b_{i}\right)-b_{i}\right] f \chi_{6^{N} Q \backslash \frac{6}{5} R}\right)(y)\right| \\
& \lesssim \frac{1}{\lambda\left(y, r_{R}\right)} \int_{6^{N} Q \backslash \frac{6}{5} R} \prod_{i=1}^{k}\left|b_{i}(z)-m_{R}\left(b_{i}\right)\right||f(z)| d \mu(z) \\
& \lesssim\left\{\frac{1}{\lambda\left(y, r_{R}\right)} \int_{6^{N} Q} \prod_{i=1}^{k}\left|b_{i}(z)-m_{R}\left(b_{i}\right)\right|^{r^{\prime}} d \mu(z)\right\}^{\frac{1}{r^{\prime}}}\left\{\frac{1}{\lambda\left(y, r_{R}\right)} \int_{6^{N} Q}|f(z)|^{r} d \mu(z)\right\}^{\frac{1}{r}} \\
& \lesssim\|\vec{b}\| \operatorname{RBMO}(\mu) \\
& M_{r, 5} f(x) .
\end{aligned}
$$

Therefore, for all $x \in Q$,

$$
\mathrm{L}_{4} \lesssim\|\vec{b}\|_{\mathrm{RBMO}(\mu)} M_{r, 5} f(x) .
$$

Combining $\mathrm{L}_{1}, \mathrm{~L}_{2}, \mathrm{~L}_{3}$ and $\mathrm{L}_{4}$, we then obtain (3.10) and hence completes the proof of Theorem 1.9. 


\section{Proof of Theorem 1.13}

To prove Theorem 1.13, we need the following generalized Hölder inequality presented in [7, pp. 246-247] in the setting of $\mathbb{R}^{d}$ with $\mu$ as in (1.1) (see also [19, Lemmas 2.2 and 2.3] for the setting of $\mathbb{R}^{d}$ with $\mu$ being the $d$-dimensional Lebesgue measure). The proofs in $[19,7]$ also work for non-homogeneous spaces and we omit the details.

Lemma 4.1. There exists a positive constant $C$, depending only on $k$, such that, for all locally integrable functions $f$ and $b_{i}$ with $i \in\{1, \cdots, k\}$, all balls $B$ and $1 / r=1 / r_{1}+\cdots+1 / r_{k}$ with $r_{i} \in[1, \infty)$ for $i \in\{1, \cdots, k\}$,

$$
\begin{aligned}
& \frac{1}{\mu(2 B)} \int_{B}\left|f(x) b_{1}(x) \cdots b_{k}(x)\right| d \mu(x) \\
\leq & C\left\|b_{1}\right\|_{\exp L^{r_{1}}, B, \frac{\mu}{\mu(2 B)}} \cdots\left\|b_{k}\right\|_{\exp L^{r} k, B, \frac{\mu}{\mu(2 B)}}\|f\|_{L(\log L)^{1 / r}, B, \frac{\mu}{\mu(2 B)},}
\end{aligned}
$$

where, for $\alpha \in(0, \infty)$

$$
\begin{aligned}
& \|f\|_{L(\log L)^{\alpha}, B, \frac{\mu}{\mu(2 B)}} \\
:= & \inf \left\{\lambda \in(0, \infty): \frac{1}{\mu(2 B)} \int_{B} \frac{|f(x)|}{\lambda} \log ^{\alpha}\left(2+\frac{|f(x)|}{\lambda}\right) d \mu(x) \leq 1\right\}
\end{aligned}
$$

and

$$
\|f\|_{\exp L^{\alpha}, B, \frac{\mu}{\mu(2 B)}}:=\inf \left\{\lambda \in(0, \infty): \frac{1}{\mu(2 B)} \int_{B} \exp \left(\frac{|f(x)|}{\lambda}\right)^{\alpha} d \mu(x) \leq 2\right\} .
$$

Now we are ready to prove Theorem 1.13. In what follows, for any $k \in \mathbb{N}$ and $i \in\{1, \cdots, k\}$, let $C_{i}^{k}$ be as in Section 3. For all $k$-tuples $r:=\left(r_{1}, \cdots, r_{k}\right)$ and $\sigma:=\{\sigma(1), \cdots, \sigma(i)\} \in C_{i}^{k}$, let $\vec{b}_{\sigma}:=\left(b_{\sigma(1)}, \cdots, b_{\sigma(i)}\right)$,

$$
\left\|\vec{b}_{\sigma}\right\|_{\mathrm{Osc}_{\exp L^{r}(\mu)}}:=\left\|b_{\sigma(1)}\right\|_{\mathrm{Osc}_{\exp L^{r} \sigma(1)}(\mu)} \cdots\left\|b_{\sigma(i)}\right\|_{\mathrm{Osc}_{\exp L^{r} \sigma(i)}(\mu)}
$$

and, for a finite family $\vec{b}:=\left(b_{1}, b_{2}, \cdots, b_{k}\right)$ of locally integrable functions, let

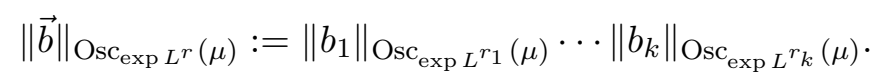

Proof of Theorem 1.13. Similar to the proof of [7, Theorem 4], without loss of generality, we may assume that, for all $i \in\{1, \cdots, k\},\left\|b_{i}\right\|_{\mathrm{Osc}_{\exp L^{r^{i}}}(\mu)}=1$.

We prove the theorem in two cases: $k=1$ and $k>1$.

Case I. $k=1$. For any given bounded function $f$ with bounded support and any $\lambda>\beta_{6}\|f\|_{L^{1}(\mu)} /\|\mu\|$, applying the Calderón-Zygmund decomposition to $f$ at level $\lambda$ (see [2, Theorem 6.3]), we see that, with the same notation as in [2, Theorem 6.3], $f=g+h$, where $g:=f \chi_{\mathcal{X} \backslash \cup_{j} 6 Q_{j}}+\sum_{j} \varphi_{j}, h:=\sum_{j}\left(\omega_{j} f-\varphi_{j}\right)$, and 
(a) $\left\{Q_{j}\right\}_{j}$ is a sequence of pairwise disjoint balls in $\mathcal{X}$ and $\left\{6 Q_{j}\right\}_{j}$ is finite overlapped;

(b) $|f(x)| \leq \lambda$ for $\mu$-almost every $x \in \mathcal{X} \backslash \cup_{j} 6 Q_{j}$;

(c) $\frac{\lambda}{\beta_{6}}<\frac{1}{\mu\left(6^{2} Q_{j}\right)} \int_{Q_{j}}|f(x)| d \mu(x)$;

(d) $\left\{\varphi_{j}\right\}_{j}$ is a family of functions satisfying that $\operatorname{supp} \varphi_{j} \subset R_{j}$ and

$$
\int_{R_{j}} \varphi_{j}(x) d \mu(x)=\int_{6 Q_{j}} f(x) \omega_{j}(x) d \mu(x),
$$

where $R_{j}$ is the smallest $\left(3 \times 6^{2}, C_{\lambda}^{\log _{2}\left(3 \times 6^{2}\right)+1}\right)$-doubling ball of the form $(3 \times$ $\left.6^{2}\right)^{k} Q_{j}$ with $k \in \mathbb{N}$ and $\omega_{j}(x):=\frac{\chi_{6 Q_{j}}(x)}{\sum_{i} \chi_{6 Q_{i}}(x)}$ for all $x \in \mathcal{X} ;$

(e) $\sum_{j}\left|\varphi_{j}(x)\right| \lesssim \lambda$ for $\mu$-almost every $x \in \mathcal{X}$;

(f) $\left\|\varphi_{j}\right\|_{L^{\infty}(\mu)} \mu\left(R_{j}\right) \lesssim \int_{\mathcal{X}}|f(x)| \omega_{j}(x) d \mu$.

By (c), (d) and (e), we easily know that $|g(x)| \lesssim \lambda$ for $\mu$-almost every $x \in \mathcal{X}$ and $\|g\|_{L^{2}(\mu)}^{2} \lesssim \lambda\|f\|_{L^{1}(\mu)}$, which, together with Theorem 3.10, further implies that

$\mu\left(\left\{x \in \mathcal{X}:\left|T_{b} g(x)\right|>\lambda\right\}\right) \lesssim \lambda^{-2}\left\|T_{b} g\right\|_{L^{2}(\mu)}^{2} \lesssim \lambda^{-2}\|g\|_{L^{2}(\mu)}^{2} \lesssim \lambda^{-1} \int_{\mathcal{X}}|f(y)| d \mu(y)$,

where $T_{b}:=T_{b_{1}}$. On the other hand, by (c) and (a), we see that

$$
\mu\left(\bigcup_{j} 6^{2} Q_{j}\right) \lesssim \frac{1}{\lambda} \int_{\mathcal{X}}|f(x)| d \mu(x) .
$$

Therefore, the proof of Theorem 1.13 in Case I can be reduced to proving that

$$
\mu\left(\left\{x \in \mathcal{X} \backslash \cup_{j} 6^{2} Q_{j}:\left|T_{b} h(x)\right|>\lambda\right\}\right) \lesssim \int_{\mathcal{X}} \frac{|f(y)|}{\lambda} \log ^{1 / r}\left(2+\frac{|f(y)|}{\lambda}\right) d \mu(y) .
$$

To see this, for all $j$ and $x \in \mathcal{X}$, we let $b_{j}(x):=b(x)-m_{\widetilde{Q}_{j}}(b)$ and

$$
h_{j}(x):=\omega_{j}(x) f(x)-\varphi_{j}(x)
$$

and write

$$
T_{b} h(x)=\sum_{j} b_{j}(x) T h_{j}(x)-\sum_{j} T\left(b_{j} h_{j}\right)(x)=: \mathrm{I}(x)+\mathrm{II}(x) .
$$

For the term $\operatorname{II}(x)$, by [2, Theorem 6.5], we know that $T$ is of weak type $(1,1)$ and hence 


$$
\begin{aligned}
& \mu(\{x \in \mathcal{X}:|\operatorname{II}(x)|>\lambda\}) \\
\lesssim & \lambda^{-1} \sum_{j} \int_{\mathcal{X}}\left|b_{j}(y) h_{j}(y)\right| d \mu(y) \\
\lesssim & \lambda^{-1} \sum_{j} \int_{6 Q_{j}}\left|b(y)-m_{\widetilde{Q}_{j}}(b)\right||f(y)| \omega_{j}(y) d \mu(y) \\
& +\lambda^{-1} \sum_{j}\left\|\varphi_{j}\right\|_{L^{\infty}(\mu)} \int_{R_{j}}\left|b(y)-m_{\widetilde{Q}_{j}}(b)\right| d \mu(y)=: \mathrm{E}+\mathrm{F} .
\end{aligned}
$$

Obviously, $R_{j}$ is $\left(6, \beta_{6}\right)$-doubling and hence $R_{j}=\widetilde{R}_{j}$. Thus, it follows, from Lemma 3.2 , that

$$
\begin{aligned}
& \int_{R_{j}}\left|b(y)-m_{\widetilde{Q_{j}}}(b)\right| d \mu(y) \\
\leq & \int_{R_{j}}\left|b(y)-m_{R_{j}}(b)\right| d \mu(y)+\mu\left(R_{j}\right)\left[\left|m_{\widetilde{6 Q_{j}}}(b)-m_{R_{j}}(b)\right|+\left|m_{\widetilde{6 Q_{j}}}(b)-m_{\widetilde{Q_{j}}}(b)\right|\right] \\
\lesssim & \mu\left(6 R_{j}\right)+\mu\left(R_{j}\right)\left[\delta\left(6 Q_{j}, R_{j}\right)+\delta\left(Q_{j}, 6 Q_{j}\right)\right] .
\end{aligned}
$$

By Lemma 3.1, we have $\delta\left(6 Q_{j}, R_{j}\right) \lesssim 1$ and $\delta\left(Q_{j}, 6 Q_{j}\right) \lesssim 1$, which, together with $\mu\left(6 R_{j}\right) \leq \beta_{6} \mu\left(R_{j}\right)$, further implies that

$$
\mathrm{F} \lesssim \lambda^{-1} \sum_{j}\left\|\varphi_{j}\right\|_{L^{\infty}(\mu)} \mu\left(R_{j}\right) \lesssim \lambda^{-1} \int_{\mathcal{X}}|f(y)| d \mu(y) .
$$

On the other hand, from Lemma 4.1, $\left\|b_{j}\right\|_{\mathrm{Osc}_{\exp L^{r}}(\mu)}=1$ for $j \in\{1, \cdots, k\}$, (c) and (d), it follows that

$$
\begin{aligned}
\mathrm{E} & \lesssim \lambda^{-1} \sum_{j} \mu\left(12 Q_{j}\right)\left\|f \omega_{j}\right\|_{L(\log L)^{1 / r}, 6 Q_{j}, \mu / \mu\left(12 Q_{j}\right)}\left\|b_{j}\right\|_{\exp L^{r}, 6 Q_{j}, \mu / \mu\left(12 Q_{j}\right)} \\
& \lesssim \lambda^{-1} \sum_{j} \mu\left(12 Q_{j}\right)\left\|f \omega_{j}\right\|_{L(\log L)^{1 / r}, 6 Q_{j}, \mu / \mu\left(12 Q_{j}\right)} \\
\lesssim & \lambda^{-1} \sum_{j} \mu\left(12 Q_{j}\right) \\
& \times \inf _{t \in(0, \infty)}\left\{t+\frac{t}{\mu\left(12 Q_{j}\right)} \int_{6 Q_{j}} \frac{|f(y)| \omega_{j}(y)}{t} \log ^{1 / r}\left(2+\frac{|f(y)| \omega_{j}(y)}{t}\right) d \mu(y)\right\} \\
\lesssim & \int_{\mathcal{X}} \frac{|f(y)|}{\lambda} \log ^{1 / r}\left(2+\frac{|f(x)|}{\lambda}\right) d \mu(y) .
\end{aligned}
$$

Thus, we conclude that

$$
\mu(\{x \in \mathcal{X}:|\mathrm{II}(x)|>\lambda\}) \lesssim \int_{\mathcal{X}} \frac{|f(y)|}{\lambda} \log ^{1 / r}\left(2+\frac{|f(y)|}{\lambda}\right) d \mu(y) .
$$


Now we turn to $\mathrm{I}(x)$. Let $x_{j}$ be the center of $Q_{j}$. Since supp $h_{j} \subset R_{j}$, for $x \in \mathcal{X} \backslash 2 R_{j}$, using (1.9) and $\int_{\mathcal{X}} b_{j}(x) d \mu(x)=0$, we write

$$
\begin{aligned}
& \int_{\mathcal{X} \backslash \cup_{j} 6^{2} Q_{j}}|\mathrm{I}(x)| d \mu(x) \\
\lesssim & \sum_{j} r_{R_{j}}^{\delta} \int_{\mathcal{X}}\left|h_{j}(y)\right| d \mu(y) \int_{\mathcal{X} \backslash 2 R_{j}} \frac{\left|b_{j}(x)\right|}{d\left(x, x_{j}\right)^{\delta} \lambda\left(x_{j}, d\left(x, x_{j}\right)\right)} d \mu(x) \\
& +\sum_{j} \int_{2 R_{j} \backslash 6^{2} Q_{j}}\left|b_{j}(x)\right|\left|T\left(\omega_{j} f\right)(x)\right| d \mu(x) \\
& +\sum_{j} \int_{2 R_{j}}\left|b_{j}(x)\right|\left|T\left(\varphi_{j}\right)(x)\right| d \mu(x)=: \mathrm{G}+\mathrm{H}+\mathrm{J}
\end{aligned}
$$

Using (1.3), Lemmas 3.1 and 3.2, we see that

$$
\begin{aligned}
& \int_{\mathcal{X} \backslash 2 R_{j}} \frac{\left|b_{j}(x)\right|}{d\left(x, x_{j}\right)^{\delta} \lambda\left(x_{j}, d\left(x, x_{j}\right)\right)} d \mu(x) \\
& \lesssim \sum_{k=1}^{\infty}\left(2^{k} r_{R_{j}}\right)^{-\delta} \frac{1}{\lambda\left(x_{j}, 2^{k} r_{R_{j}}\right)} \int_{2^{k+1} R_{j}}\left|b(x)-m_{2^{k+1} R_{j}}(b)\right| d \mu(x) \\
& +\sum_{k=1}^{\infty}\left(2^{k} r_{R_{j}}\right)^{-\delta} \frac{\mu\left(2^{k+1} R_{j}\right)}{\lambda\left(x_{j}, 2^{k} r_{R_{j}}\right)}\left|m_{\widetilde{Q_{j}}}(b)-m_{2^{k+1} R_{j}}(b)\right| \\
& \lesssim \sum_{k=1}^{\infty}\left(2^{k} r_{R_{j}}\right)^{-\delta} \frac{\mu\left(2^{k+2} R_{j}\right)}{\lambda\left(x_{j}, 2^{k} r_{R_{j}}\right)} \\
& +\sum_{k=1}^{\infty} \delta\left(\widetilde{Q_{j}}, \widetilde{2^{k+1} R_{j}}\right)\left(2^{k} r_{R_{j}}\right)^{-\delta} \frac{\mu\left(2^{k+1} R_{j}\right)}{\lambda\left(x_{j}, 2^{k} r_{R_{j}}\right)} \lesssim r_{R_{j}}^{-\delta},
\end{aligned}
$$

where the last inequality follows from an argument similar to $I_{3}$. From this, together with

$$
\left\|h_{j}\right\|_{L^{1}(\mu)} \lesssim \int_{\mathcal{X}}|f(y)| \omega_{j}(y) d \mu(y)
$$

we then infer that

$$
\mathrm{G} \lesssim \int_{\mathcal{X}}|f(y)| d \mu(y)
$$

On the other hand, by Hölder's inequality, the $L^{2}(\mu)$-boundedness of $T$, Lemma 3.2 , the fact that $R_{j}$ is doubling and (f), we conclude that

$$
\mathrm{J} \leq \sum_{j} \int_{2 R_{j}}\left|b(x)-m_{\widetilde{2 R_{j}}}(b)\right|\left|T\left(\varphi_{j}\right)(x)\right| d \mu(x)
$$




$$
\begin{aligned}
& +\sum_{j}\left|m_{\widetilde{Q_{j}}}(b)-m_{\widetilde{2 R_{j}}}(b)\right| \int_{2 R_{j}}\left|T\left(\varphi_{j}\right)(x)\right| d \mu(x) \\
\leq & \sum_{j}\left(\int_{2 R_{j}}\left|b(x)-m_{\widetilde{2 R_{j}}}(b)\right|^{2} d \mu(x)\right)^{1 / 2}\left\|T \varphi_{j}\right\|_{L^{2}(\mu)} \\
& +\sum_{j}\left[\mu\left(2 R_{j}\right)\right]^{1 / 2}\left\|T \varphi_{j}\right\|_{L^{2}(\mu)} \times\left|m_{\widetilde{Q_{j}}}(b)-m_{\widetilde{2 R_{j}}}(b)\right| \\
\lesssim & \sum_{j}\left[\mu\left(4 R_{j}\right)\right]^{1 / 2}\left\|T \varphi_{j}\right\|_{L^{2}(\mu)}\left[1+\left|m_{\widetilde{Q_{j}}}(b)-m_{\widetilde{2 R_{j}}}(b)\right|\right] \\
\lesssim & \sum_{j}\left[\mu\left(4 R_{j}\right)\right]^{1 / 2}\left\|T \varphi_{j}\right\|_{L^{2}(\mu)} \lesssim \int_{\mathcal{X}}|f(y)| d \mu(y),
\end{aligned}
$$

where the second to the last inequality follows from the fact that

$$
\begin{aligned}
& \left|m_{\widetilde{Q_{j}}}(b)-m_{\widetilde{2 R_{j}}}(b)\right| \\
\leq & \left|m_{\widetilde{Q_{j}}}(b)-m_{R_{j}}(b)\right|+\left|m_{R_{j}}(b)-m_{2 R_{j}}(b)\right|+\left|m_{2 R_{j}}(b)-m_{\widetilde{2 R_{j}}}(b)\right| \lesssim 1 .
\end{aligned}
$$

To estimate $\mathrm{H}$, observe that by (1.8), for all $x \in 2 R_{j} \backslash 6^{2} Q_{j}$,

$$
\left|T\left(\omega_{j} f\right)(x)\right| \lesssim \frac{1}{\lambda\left(x_{j}, d\left(x, x_{j}\right)\right)} \int_{\mathcal{X}} \omega_{j}(y)|f(y)| d \mu(y) .
$$

Therefore, by Lemma 3.2,

$$
\begin{aligned}
\mathrm{H} \lesssim & \sum_{j}\left\{\int_{2 R_{j} \backslash R_{j}} \frac{\left|b_{j}(x)\right|}{\lambda\left(x_{j}, d\left(x, x_{j}\right)\right)} d \mu(x)+\int_{R_{j} \backslash Q_{j}} \frac{\left|b_{j}(x)\right|}{\lambda\left(x_{j}, d\left(x, x_{j}\right)\right)} d \mu(x)\right\} \\
& \times \int_{\mathcal{X}} \omega_{j}(y)|f(y)| d \mu(y) \\
\lesssim & \sum_{j}\left\{\frac{\mu\left(4 R_{j}\right)}{\lambda\left(x_{j}, r_{R_{j}}\right)}+\frac{\mu\left(2 R_{j}\right)}{\lambda\left(x_{j}, r_{R_{j}}\right)}\right\} \int_{\mathcal{X}} \omega_{j}(y)|f(y)| d \mu(y) \\
& +\sum_{j} \sum_{k=0}^{N-1} \frac{1}{\lambda\left(x_{j},\left(3 \times 6^{2}\right)^{k} r_{Q_{j}}\right)} \int_{\left(3 \times 6^{2}\right)^{k+1} Q_{j} \backslash\left(3 \times 6^{2}\right)^{k} Q_{j}}\left|b(x)-m_{\left(3 \times \widetilde{\left.6^{2}\right)^{k+1}} Q_{j}\right.}(b)\right| d \mu(x) \\
& \times \int_{\mathcal{X}} \omega_{j}(y)|f(y)| d \mu(y) \\
& +\sum_{j} \sum_{k=0}^{N-1} \frac{\mu\left(\left(3 \times 6^{2}\right)^{k+1} Q_{j}\right)}{\lambda\left(x_{j},\left(3 \times 6^{2}\right)^{k} r_{Q_{j}}\right)}\left|m_{\widetilde{Q_{j}}}(b)-m_{\left(3 \times \widetilde{\left.6^{2}\right)^{k+1}} Q_{j}\right.}(b)\right| \int_{\mathcal{X}} \omega_{j}(y)|f(y)| d \mu(y),
\end{aligned}
$$

where $N$ satisfies $R_{j}=\left(3 \times 6^{2}\right)^{N} Q_{j}$. Obviously, for all $k \in\{0, \cdots, N-1\}$, $\left(3 \times 6^{2}\right)^{k} Q_{j} \subset R_{j}$ and so

$$
\left|m_{\widetilde{Q}_{j}}(b)-m_{\left(3 \times 6^{2}\right)^{k+1} Q_{j}}(b)\right| \lesssim \delta\left(Q_{j},\left(3 \times 6^{2}\right)^{k+1} Q_{j}\right) \lesssim \delta\left(Q_{j}, R_{j}\right) \lesssim 1 .
$$


Consequently, by the fact that $R_{j}$ is the smallest $\left(3 \times 6^{2}, C_{\lambda}^{\log _{2}\left(3 \times 6^{2}\right)+1}\right)$-doubling ball of type $\left(3 \times 6^{2}\right)^{i} Q_{j}$ with $i \in \mathbb{N},(1.3)$, Lemma 3.2 and an argument similar to that used in the proof of Lemma 3.5(iii), together with (d), we see that

$$
\begin{aligned}
\mathrm{H} \lesssim \sum_{j} \int_{\mathcal{X}} \omega_{j}(y)|f(y)| d \mu(y)+\sum_{j} \sum_{k=0}^{N-1} \frac{\mu\left(\left(3 \times 6^{2}\right)^{k+2} Q_{j}\right)}{\lambda\left(x_{j},\left(3 \times 6^{2}\right)^{k} r_{Q_{j}}\right)} \int_{\mathcal{X}} \omega_{j}(y)|f(y)| d \mu(y) \\
\quad+\sum_{j} \sum_{k=0}^{N-1} \frac{\mu\left(\left(3 \times 6^{2}\right)^{k+1} Q_{j}\right)}{\lambda\left(x_{j},\left(3 \times 6^{2}\right)^{k} r_{Q_{j}}\right)} \int_{\mathcal{X}} \omega_{j}(y)|f(y)| d \mu(y) \\
\lesssim \sum_{j} \int_{\mathcal{X}} \omega_{j}(y)|f(y)| d \mu(y)+\sum_{j} \sum_{k=0}^{N-1} \frac{\mu\left(\left(3 \times 6^{2}\right)^{k} Q_{j}\right)}{\lambda\left(x_{j},\left(3 \times 6^{2}\right)^{k} r_{Q_{j}}\right)} \int_{\mathcal{X}} \omega_{j}(y)|f(y)| d \mu(y) \\
\lesssim \int_{\mathcal{X}}|f(y)| d \mu(y) .
\end{aligned}
$$

Combining the estimates for $\mathrm{G}, \mathrm{H}$ and $\mathrm{J}$ above, we then conclude that

$$
\int_{\mathcal{X} \backslash \cup_{j} 6^{2} Q_{j}}|\mathrm{I}(x)| d \mu(x) \lesssim \int_{\mathcal{X}}|f(y)| d \mu(y),
$$

which implies the desired conclusion and hence completes the proof of Theorem 1.13 in the case that $k=1$.

Case II. $k \geq 2$. The proof of this case is completely similar to that of [7, Theorem 4]. We omit the details, which completes the proof of Theorem 1.13.

\section{ACKNOWLEDGMENTS}

The first author would like to thank Professors Yan Meng and Dongyong Yang for some helpful discussions on the subject of this paper. The authors also wish to express their thanks to the referee for her/his very carefully reading and also her/his so many careful, valuable and suggestive remarks which improve the presentation of this article.

\section{REFERENCES}

1. Z. Birnbaum and W. Orlicz, Über die verallgemeinerung des begriffes der zueinander konjugierten potenzen, Studia Math., 3 (1931), 1-67.

2. T. A. Bui and X. T. Duong, Hardy spaces, regularized BMO spaces and the boundedness of Calderón-Zygmund operators on non-homogeneous spaces, J. Geom. Anal., DOI 10.1007/s12220-011-9268-y (to appear, arXiv: 1009.1274).

3. W. Chen, Y. Meng and D. Yang, Calderon-Zygmund operators on Hardy spaces without the doubling condition, Proc. Amer. Math. Soc., 133 (2005), 2671-2680. 
4. R. R. Coifman, R. Rochberg and G. Weiss, Factorization theorems for Hardy spaces in several variables, Ann. of Math. (2), 103 (1976), 611-635.

5. R. R. Coifman and G. Weiss, Analyse Harmonique Non-Commutative sur Certains Espaces Homogènes, Lecture Notes in Math., 242, Springer-Verlag, Berlin-New York, 1971.

6. R. R. Coifman and G. Weiss, Extensions of Hardy spaces and their use in analysis, Bull. Amer. Math. Soc., 83 (1977), 569-645.

7. G. Hu, Y. Meng and D. Yang, Multilinear commutators of singular integrals with non doubling measures, Integral Equations Operator Theory, 51 (2005), 235-255.

8. G. Hu, Y. Meng and D. Yang, New atomic characterization of $H^{1}$ space with nondoubling measures and its applications, Math. Proc. Cambridge Philos. Soc., 138 (2005), 151-171.

9. T. Hytönen, A framework for non-homogeneous analysis on metric spaces, and the RBMO space of Tolsa, Publ. Mat., 54 (2010), 485-504.

10. T. Hytönen, S. Liu, Da. Yang and Do. Yang, Boundedness of Calderón-Zygmund operators on non-homogeneous metric measure spaces, Canad. J. Math., DOI 10.4153/CJM2011-065-2 (to appear, arXiv: 1011.2937).

11. T. Hytönen and H. Martikainen, Non-homogeneous $T b$ theorem and random dyadic cubes on metric measure spaces, J. Geom. Anal., DOI 10.1007/s12220-011-9230-z (to appear, arXiv: 0911.4387).

12. T. Hytönen, Da. Yang and Do. Yang, The Hardy space $H^{1}$ on non-homogeneous metric spaces, Math. Proc. Cambridge Philos. Soc., DOI 10.1017/S0305004111000776 (to appear, arXiv: 1008.3831).

13. E. Lieb and M. Loss, Analysis, American Mathematical Society, Providence, RI, 2001.

14. H. Lin and D. Yang, Spaces of type BLO on non-homogeneous metric measure spaces, Front. Math. China, 6 (2011), 271-292.

15. S. Liu, Da. Yang and Do. Yang, Boundedness of Calderón-Zygmund operators on non-homogeneous metric measure spaces: Equivalent characterizations, J. Math. Anal. Appl., 386 (2012), 258-272.

16. L. Maligranda, Indices and interpolation, Dissertationes Math. (Rozprawy Mat.), 234 (1985), 49 pp.

17. F. Nazarov, S. Treil and A. Volberg, The $T b$-theorem on non-homogeneous spaces, Acta Math., 190 (2003), 151-239.

18. W. Orlicz, Über eine gewisse Klasse von Räumen vom Typus B, Bull. Inst. Acad. Pol. Ser. $A, 8$ (1932), 207-220.

19. C. Pérez and R. Trujillo-González, Sharp weighted estimates for multilinear commutators, J. London Math. Soc. (2), 65 (2002), 672-692.

20. M. Rao and Z. Ren, Theory of Orlicz Spaces, Marcel Dekker, New York, 1991. 
21. M. Rao and Z. Ren, Applications of Orlicz Spaces, Marcel Dekker, Inc., New York, 2002.

22. X. Tolsa, BMO, $H^{1}$, and Calderón-Zygmund operators for non doubling measures, Math. Ann., 319 (2001), 89-149.

23. X. Tolsa, Littlewood-Paley theory and the $T(1)$ theorem with non-doubling measures, Adv. Math., 164 (2001), 57-116.

24. X. Tolsa, The space $H^{1}$ for nondoubling measures in terms of a grand maximal operator, Trans. Amer. Math. Soc., 355 (2003), 315-348.

25. X. Tolsa, Painlevés problem and the semiadditivity of analytic capacity, Acta Math., 190 (2003), 105-149.

26. X. Tolsa, The semiadditivity of continuous analytic capacity and the inner boundary conjecture, Amer. J. Math., 126 (2004), 523-567.

27. X. Tolsa, Bilipschitz maps, analytic capacity, and the Cauchy integral, Ann. of Math. (2), 162 (2005), 1243-1304.

28. A. Volberg and B. D. Wick, Bergman-type singular operators and the characterization of Carleson measures for Besov-Sobolev spaces on the complex ball, Amer. J. Math., (to appear, arXiv: 0910.1142).

Xing Fu, Dachun Yang and Wen Yuan

School of Mathematical Sciences

Beijing Normal University

Laboratory of Mathematics and Complex Systems

Ministry of Education

Beijing 100875

P. R. China

E-mail: xingfu@mail.bnu.edu.cn

dcyang@bnu.edu.cn

wenyuan@bnu.edu.cn 